\title{
An Analytic Framework for the Supercritical Lane-Emden Equation and its Gradient Flow
}

\section{Simon Blatt ${ }^{1}$ and Michael Struwe ${ }^{2}$}

${ }^{1}$ Fakultät für Mathematik, Karlsruher Institut für Technologie, D-76128

Karlsruhe, Germany, ${ }^{2}$ Departement Mathematik, ETH-Zürich, CH-8092

Zürich, Switzerland

Correspondence to be sent to: michael.struwe@math.ethz.ch

The natural setting for the Lane-Emden equation $-\Delta u=|u|^{p-2} u$ on a domain $\Omega \subset \mathbb{R}^{n}$, $n \geq 3$, for supercritical exponents $p>2^{*}=2 n /(n-2)$ is identified as the space of functions $u \in H_{0}^{1} \cap L^{p}(\Omega)$ with finite scale-invariant Morrey norms. We show that this Morrey regularity is propagated by the heat flow associated with this equation, and we study the blow-up profiles.

\section{Introduction}

Consider the Dirichlet problem for the Lane-Emden equation

$$
-\Delta u=|u|^{p-2} u \text { on } \Omega, \quad u=0 \text { on } \partial \Omega
$$

where $\Omega$ is a smoothly bounded domain in $\mathbb{R}^{n}, n \geq 3$. A challenging question attributed to Paul Rabinowitz is whether for domains $\Omega$ with nontrivial topology, in particular, for domains sufficiently close to an annulus, there exists a solution $u>0$ to (1.1) for 
any $p>2$ (see [2, p. S19], and [35] for further background; see also [6-8, 13, 26, 29, 30] for partial results). Formally, solutions to (1.1) correspond to critical points of the functional

$$
E_{p}(u)=\frac{1}{2} \int_{\Omega}|\nabla u|^{2} \mathrm{~d} x-\frac{1}{p} \int_{\Omega}|u|^{p} \mathrm{~d} x, \quad u \in H_{0}^{1} \cap L^{p}(\Omega) .
$$

The purpose of the present paper is to identify a functional analytic framework for dealing with problem (1.1) in the "supercritical" case when $p>2^{*}:=2 n /(n-2)$ and to discuss the corresponding heat flow

$$
u_{t}-\Delta u=|u|^{p-2} u \text { on } \Omega \times[0, T[, \quad u=0 \text { on } \partial \Omega \times[0, T[, T \leq \infty,
$$

for given initial data $u_{0} \in H^{1} \cap L^{p}(\Omega)$ as a suitable gradient flow for $E_{p}$.

Much of our work has been inspired by Pacard's [28], where he presents a remarkable monotonicity formula and a partial regularity theory for "stationary" solutions to (1.1), the notion of "stationary" solution being modeled on Evans' [9] notion of a stationary weakly harmonic map. In Proposition 5.1, moreover, we extend Pacard's [28] partial regularity result to the full range of exponents $p>2^{*}$.

Pacard's work motivates the study of (1.1) in the Morrey space $H_{0}^{1} \cap L^{p, \mu}(\Omega)$ defined below, where $\mu=\frac{2 p}{p-2}$. By combining Pacard's ideas with the arguments giving monotonicity and partial regularity for the heat flow of harmonic maps developed in [34], we obtain a monotonicity formula similar to Pacard's for the flow (1.2) from which we deduce Morrey estimates and partial regularity results. Our Proposition 6.2 shows that-up to a constant-the Morrey $L^{p, \mu}$-bounds of the data are preserved along the flow, even when the solution to (1.2) blows up in finite time. In the latter case, the Morrey estimates hold on domains whose size naturally decreases as we approach the blow-up time, which is sufficient to recover partially regular blow-up profiles as "tangent cones" to the flow by rescaling the solution suitably around blow-up points; see Theorem 6.9. The Pacard-type monotonicity formula in Proposition 3.1 in Section 3 and the $\varepsilon$-regularity result for weak solutions of (1.2), Proposition 4.1 in Section 4, also may be of interest in themselves.

A particular consequence of our estimates is that classical solutions of (1.2) on a convex domain always either blow up in finite time or uniformly decay to 0 as $t \rightarrow \infty$, as shown in Proposition 6.6, which agrees with results of Matano and Merle [22] for global radially symmetric solutions of (1.2). 
For so-called "borderline solutions" $u \geq 0$ of (1.2) in the sense of [27] partial regularity results similar to ours previously were obtained by Chou et al. [5], however, exploiting the additional global bounds available in that case.

\section{Morrey Spaces}

Recall that (in Adams' [1] notation) a function $f \in L^{p}(\Omega)$ on a domain $\Omega \subset \mathbb{R}^{n}$ belongs to the Morrey space $L^{p, \lambda}(\Omega)$ if

$$
\|f\|_{L^{p, \lambda}(\Omega)}^{p}:=\sup _{x_{0} \in R^{n}, r>0} r^{\lambda-n} \int_{B_{r}\left(x_{0}\right) \cap \Omega}|f|^{p} \mathrm{~d} x<\infty,
$$

where $B_{r}\left(x_{0}\right)$ denotes the Euclidean ball of radius $r>0$ centered at $x_{0}$.

Similarly, a function $f \in L^{p}(E)$ on a subset $E$ of the spacetime $\mathbb{R}^{n+1}$ belongs to the parabolic Morrey space $L^{p, \lambda}(E)$ if

$$
\|f\|_{L^{p, \lambda}(E)}^{p}:=\sup _{z_{0}=\left(x_{0}, t_{0}\right) \in R^{n+1}, r>0} r^{\lambda-(n+2)} \int_{P_{r}\left(z_{0}\right) \cap E}|f|^{p} \mathrm{~d} z<\infty,
$$

where $P_{r}(x, t)$ denotes the backward parabolic cylinder $\left.B_{r}(x) \times\right] t-r^{2}, t[$.

For either problem (1.1) or (1.2) the number $\mu=\frac{2 p}{p-2}$ turns out to be the relevant Morrey exponent. In fact, by work of Adams [1] for $u \in H_{0}^{1}(\Omega)$ satisfying $\nabla u \in L^{2, \mu}(\Omega)$ we have $u \in L^{p, \mu}(\Omega)$ with continuous embedding; similarly, for any $u \in W^{2, \frac{p}{p-1}} \cap H_{0}^{1}(\Omega)$ with $\Delta u \in L^{\frac{p}{p-1}, \mu}(\Omega)$ there holds $\nabla u \in L^{2, \mu}(\Omega)$ and $\|\nabla u\|_{L^{2, \mu}(\Omega)} \leq C\|\Delta u\|_{L^{\frac{p}{p-1}, \mu}(\Omega)}$. We revisit these estimates in Section 5, which, in particular, imply a threshold result for weak solutions $u \in H_{0}^{1} \cap L^{p, \mu}(\Omega)$ of (1.1); see Proposition 5.7. As we shall see, similar local and global threshold results hold true for blow-up of the heat flow (1.2).

\section{The Heat Flow}

Let $\Omega$ be a smoothly bounded domain in $\mathbb{R}^{n}, n \geq 3$, and let $u_{0} \in C_{0}^{\infty}(\Omega)$. For given $p>2$ we consider the flow (1.2) with initial data

$$
u=u_{0} \quad \text { at } t=0 .
$$

Given a smooth solution $u$ to (1.2), (3.1), upon multiplying (1.2) by $u_{t}$ and integrating by parts, for any $T>0$ we obtain the familiar energy identity

$$
E(u(T))+\int_{0}^{T} \int_{\Omega}\left|u_{t}\right|^{2} \mathrm{~d} x \mathrm{~d} t=E\left(u_{0}\right)
$$


for $E=E_{p}$. In particular, the function $t \mapsto E(u(t))$ is nonincreasing; in fact, the flow (1.2), (3.1) defines the $L^{2}$-gradient flow for $E$.

For "small" data $u_{0} \in C^{1}(\bar{\Omega})$ it is not hard to show that the solution to (1.2), (3.1) exists for all time. On the other hand, if $E\left(u_{0}\right)<0$, the flow (1.2), (3.1) will blow up in finite time $T>0$ in the sense that $\|u(t)\|_{L^{\infty}(\Omega)} \rightarrow \infty$ as $t \uparrow T$; see, for instance, Fujita [11] or Kaplan [19]. Given $0 \leq u_{0} \in C^{1}(\bar{\Omega}) \backslash\{0\}$, thus the Cauchy problem for (1.2) with initial data $\lambda u_{0}$ will have a global classical solution $u^{\lambda}$ for small $\lambda>0$, and $u^{\lambda}$ will blow up in finite time when $\lambda>0$ is large. Letting $\lambda^{*}=\sup \left\{\lambda ; u^{\lambda}\right.$ is global $\}$, one may hope that on a suitable domain for suitable data $u_{0}$ the "borderline" solution $u^{*}=\lim _{\lambda \uparrow \lambda^{*}} u^{\lambda}$ introduced by $\mathrm{Ni}$ et al. [27] will converge, as $t \rightarrow \infty$ to a solution $u_{\infty}>0$ of the time-independent problem (1.1). However, $\lambda^{*}$ might be smaller than the number where $\sup _{\lambda>0} E\left(\lambda u_{0}\right)$ is achieved, which sparks our interest also in solutions to (1.2) blowing up in finite time.

\subsection{The Giga-Kohn analysis}

In a seminal paper, Giga and Kohn [15] studied the asymptotic behavior of bounded solutions to (1.2) on the space-time cylinder $\left.P_{1}:=B_{1}(0) \times\right]-1,0\left[\subset \mathbb{R}^{n} \times \mathbb{R}\right.$ blowing up at the origin of space-time. Note that a solution $u$ to (1.2) on $P_{1}$ induces a family

$$
u_{R}(x, t)=R^{2 /(p-2)} u\left(R x, R^{2} t\right), \quad R>0
$$

of solutions to this equation on the scaled domains $\left.P_{1 / R}=B_{1 / R}(0) \times\right]-1 / R^{2}$, 0 [. In the case when $n \geq 3, p \leq 2^{*}$, and when

$$
|t|^{1 /(p-2)}|u(x, t)| \leq C \quad \text { uniformly on } P_{1},
$$

Giga-Kohn were able to derive a precise characterization of the blow-up profile, improving previous results of Weissler [37] and Friedman and McLeod [10]. Their technique relies on the introduction of self-similar variables

$$
s=-\log (-t), \quad y=x / \sqrt{-t}, \quad w(y, s)=(-t)^{\beta} u(x, t)=\mathrm{e}^{-\beta s} u\left(\mathrm{e}^{-s / 2} y,-\mathrm{e}^{-s}\right),
$$

where $\beta=1 /(p-2)$. The function $w$ then satisfies the equation

$$
w_{s}-\Delta w+\frac{1}{2} y \cdot \nabla w+\beta w=|w|^{p-2} w
$$

and an energy-type inequality for solutions of (3.6) allows one to conclude that the functions $w(\cdot, s)$ as $s \rightarrow \pm \infty$ converge to limits $w^{ \pm}$independent of $s$, corresponding to 
self-similar solutions $u^{ \pm} \equiv u_{R}^{ \pm}$for any $R>0$ of (1.2) on $\left.\mathbb{R}^{n} \times\right]-\infty, 0[$, much in the same way as blowing up a minimal surface around a singular point produces a tangent cone. The paper [15] thus provides the first instance of a "monotonicity formula" being employed in the analysis of a nonlinear flow problem. Later, analogous monotonicity formulas were discovered also for the harmonic map heat flow by Struwe [34] and for the mean curvature flow by Huisken [18]. Ultimately, monotonicity formulas for the Ricci flow played a key role in Perelman's [31] proof of the Poincaré conjecture.

In the latter geometric applications, monotonicity formulas either involve the Dirichlet energy, the area, or curvature; in contrast, the quantity in the Giga-Kohn monotonicity formula is the difference of two non-negative terms related to energy and is noncoercive.

\subsection{Pacard-type monotonicity}

Our first goal in this paper is to develop an improved monotonicity formula for the flow (1.2), similar to the monotonicity formula found by Pacard [28] for the time-independent problem. We achieve this through a combination of Pacard's ideas with the approach to the heat flow of harmonic maps presented in [34].

Let $\Omega$ be a smoothly bounded domain in $\mathbb{R}^{n}, n \geq 3$. Fix a point $x_{0} \in \Omega$ and a time $t_{0}>0$. Given a solution $u$ to (1.2), for $R>0$ let

$$
u_{R}(x, t)=R^{2 /(p-2)} u\left(x_{0}+R x, t_{0}+R^{2} t\right) \quad \text { on } \Omega_{R} \times\left[-\frac{t_{0}}{R^{2}}, 0[\right.
$$

similar to (3.3), where $\Omega_{R}=\left\{x ; x_{0}+R x \in \Omega\right\}$. Also let

$$
G(x, t)=\frac{1}{(4 \pi|t|)^{n / 2}} \mathrm{e}^{-\frac{|x|^{2}}{4|t|}}, \quad x \in \mathbb{R}^{n}, t<0,
$$

be the fundamental solution to the heat equation with singularity at $(0,0)$, and set

$$
G_{\left(x_{0}, t_{0}\right)}(x, t)=G\left(x-x_{0}, t-t_{0}\right), \quad G_{*}(x)=G(x,-1)=\frac{1}{(4 \pi)^{n / 2}} \mathrm{e}^{-|x|^{2} / 4}
$$

for brevity. We sometimes also write $z=(x, t)$ for a generic point in space-time.

It will be useful to be able to work with localized quantities, in particular, when the domain $\Omega$ is nonconvex. For this purpose, let $\varphi=\varphi(|x|) \in C^{\infty}\left(\mathbb{R}^{n}\right)$ be a compactly supported cutoff function such that $0 \leq \varphi \leq 1$, and, for $R>0$, let

$$
\varphi_{R}(x)=\varphi(R x)
$$


Define

$$
\begin{aligned}
D^{\varphi}(R) & =\frac{1}{2} \int_{\Omega_{R}}\left|\nabla u_{R}(x,-1)\right|^{2} \varphi_{R}^{2}(x) G_{*}(x) \mathrm{d} x \\
& =\frac{1}{2} R^{\frac{2 p}{p-2}} \int_{\Omega \times\left\{t_{0}-R^{2}\right\}}|\nabla u|^{2} \varphi^{2}\left(x-x_{0}\right) G_{\left(x_{0}, t_{0}\right)} \mathrm{d} x .
\end{aligned}
$$

For $q \geq 2$ we also let

$$
\begin{aligned}
F_{q}^{\varphi}(R) & =\frac{1}{q} \int_{\Omega_{R}}\left|u_{R}(x,-1)\right|^{q} \varphi_{R}^{2}(x) G_{*}(x) \mathrm{d} x \\
& =\frac{1}{q} R^{\frac{2 q}{p-2}} \int_{\Omega \times\left\{t_{0}-R^{2}\right\}}|u|^{q} \varphi^{2}\left(x-x_{0}\right) G_{\left(x_{0}, t_{0}\right)} \mathrm{d} x .
\end{aligned}
$$

Observe the identities

$$
\left.\frac{\mathrm{d}}{\mathrm{d} R}\right|_{R=1} u_{R}(x,-1)=\left(x \cdot \nabla u-2 u_{t}+\frac{2}{p-2} u\right)\left(x_{0}+x, t_{0}-1\right)
$$

and

$$
\left.\frac{\mathrm{d}}{\mathrm{d} R}\right|_{R=1} \int_{\Omega_{R}} f \mathrm{~d} x=-\int_{\partial \Omega}\left(x-x_{0}\right) \cdot v f\left(x-x_{0}\right) \mathrm{d} o
$$

for any $f \in C^{0}(\bar{\Omega})$, where $v$ is the exterior unit normal along $\partial \Omega$.

Shift coordinates so that $x_{0}=0$. Then, integrating by parts and using the Equation (1.2), we compute

$$
\begin{aligned}
\left.\frac{\mathrm{d}}{\mathrm{d} R} D^{\varphi}(R)\right|_{R=1}= & \left.\int_{\Omega_{R} \times\{-1\}} \nabla u_{R} \nabla\left(\frac{\mathrm{d}}{\mathrm{d} R} u_{R}\right) \varphi_{R}^{2} G_{*} \mathrm{~d} x\right|_{R=1} \\
& +\left.\frac{1}{2} \int_{\Omega_{R} \times\{-1\}}\left|\nabla u_{R}\right|^{2} x \cdot \nabla \varphi^{2}(R x) G_{*} \mathrm{~d} x\right|_{R=1}-B^{\varphi} \\
= & \int_{\Omega \times\left\{t_{0}-1\right\}}\left(-\Delta u+\frac{1}{2} x \cdot \nabla u\right)\left(x \cdot \nabla u-2 u_{t}+\frac{2}{p-2} u\right) \varphi^{2} G_{\left(x_{0}, t_{0}\right)} \mathrm{d} x+I^{\varphi} \\
= & \frac{1}{2} \int_{\Omega \times\left\{t_{0}-1\right\}}\left(x \cdot \nabla u-2 u_{t}+\frac{2}{p-2} u\right)^{2} \varphi^{2} G_{\left(x_{0}, t_{0}\right)} \mathrm{d} x \\
& +\int_{\Omega \times\left\{t_{0}-1\right\}}\left(|u|^{p-2} u-\frac{u}{p-2}\right)\left(x \cdot \nabla u-2 u_{t}+\frac{2}{p-2} u\right) \varphi^{2} G_{\left(x_{0}, t_{0}\right)} \mathrm{d} x+I^{\varphi},
\end{aligned}
$$

with $I^{\varphi}=B^{\varphi}+L_{D}^{\varphi}$, where $B^{\varphi}$ is the boundary term

$$
B^{\varphi}=\frac{1}{2} \int_{\partial \Omega \times\left\{t_{0}-1\right\}} v \cdot x|\nabla u|^{2} \varphi^{2} G_{\left(x_{0}, t_{0}\right)} \mathrm{d} o
$$


and where $L_{D}^{\varphi}$ is the error term

$$
\begin{aligned}
L_{D}^{\varphi}= & \int_{\Omega \times\left\{t_{0}-1\right\}}|\nabla u|^{2} \varphi x \cdot \nabla \varphi G_{\left(x_{0}, t_{0}\right)} \mathrm{d} x \\
& -2 \int_{\Omega \times\left\{t_{0}-1\right\}} \nabla u \cdot \nabla \varphi\left(x \cdot \nabla u-2 u_{t}+\frac{2}{p-2} u\right) \varphi G_{\left(x_{0}, t_{0}\right)} \mathrm{d} x
\end{aligned}
$$

induced by localization. Similarly, for $q \geq 2$, we have

$$
\begin{aligned}
\left.\frac{\mathrm{d}}{\mathrm{d} R} F_{q}^{\varphi}(R)\right|_{R=1}= & \left.\int_{\Omega_{R} \times\{-1\}}\left|u_{R}\right|^{q-2} u_{R}\left(\frac{\mathrm{d}}{\mathrm{d} R} u_{R}\right) \varphi_{R}^{2} G_{*} \mathrm{~d} x\right|_{R=1} \\
& +\left.\frac{1}{q} \int_{\Omega_{R} \times\{-1\}}\left|u_{R}\right|^{q} X \cdot \nabla \varphi^{2}(R x) G_{*} \mathrm{~d} x\right|_{R=1} \\
= & \int_{\Omega \times\left\{t_{0}-1\right\}}|u|^{q-2} u\left(x \cdot \nabla u-2 u_{t}+\frac{2}{p-2} u\right) \varphi^{2} G_{\left(x_{0}, t_{0}\right)} \mathrm{d} x+L_{q}^{\varphi},
\end{aligned}
$$

where

$$
L_{q}^{\varphi}=\frac{1}{q} \int_{\Omega \times\left\{t_{0}-1\right\}}|u|^{q} X \cdot \nabla \varphi^{2} G_{\left(x_{0}, t_{0}\right)} \mathrm{d} x
$$

In particular, we can now write

$$
\begin{aligned}
\left.\frac{\mathrm{d}}{\mathrm{d} R} D^{\varphi}(R)\right|_{R=1}= & \frac{1}{2} \int_{\Omega \times\left\{t_{0}-1\right\}}\left(x \cdot \nabla u-2 u_{t}+\frac{2}{p-2} u\right)^{2} \varphi^{2} G_{\left(X_{0}, t_{0}\right)} \mathrm{d} x \\
& +\left.\frac{\mathrm{d}}{\mathrm{d} R} F_{p}^{\varphi}(R)\right|_{R=1}-\left.\frac{1}{p-2} \frac{\mathrm{d}}{\mathrm{d} R} F_{2}^{\varphi}(R)\right|_{R=1}+A_{D}^{\varphi}+B^{\varphi}
\end{aligned}
$$

where

$$
A_{D}^{\varphi}=L_{D}^{\varphi}-L_{p}^{\varphi}+\frac{1}{p-2} L_{2}^{\varphi}
$$

which is the identity corresponding to the monotonicity formula of Giga-Kohn [15, Proposition 3]. 
On the other hand, using (1.2), we find the equation

$$
\begin{aligned}
\left.\frac{\mathrm{d}}{\mathrm{d} R} F_{2}^{\varphi}(R)\right|_{R=1}= & \int_{\Omega \times\left\{t_{0}-1\right\}} u\left(x \cdot \nabla u-2 u_{t}+\frac{2}{p-2} u\right) \varphi^{2} G_{\left(x_{0}, t_{0}\right)} \mathrm{d} x+L_{2}^{\varphi} \\
= & \int_{\Omega \times\left\{t_{0}-1\right\}} u\left(x \cdot \nabla u-2 \Delta u-2|u|^{p-2} u+\frac{2}{p-2} u\right) \varphi^{2} G_{\left(x_{0}, t_{0}\right)} \mathrm{d} x+L_{2}^{\varphi} \\
= & 2 \int_{\Omega \times\left\{t_{0}-1\right\}}|\nabla u|^{2} \varphi^{2} G_{\left(x_{0}, t_{0}\right)} \mathrm{d} x \\
& -2 \int_{\Omega \times\left\{t_{0}-1\right\}} u\left(|u|^{p-2} u-\frac{u}{p-2}\right) \varphi^{2} G_{\left(x_{0}, t_{0}\right)} \mathrm{d} x+A_{2}^{\varphi} \\
= & 4 D^{\varphi}(1)-2 p F_{p}^{\varphi}(1)+\frac{4}{p-2} F_{2}^{\varphi}(1)+A_{2}^{\varphi},
\end{aligned}
$$

where

$$
A_{2}^{\varphi}=L_{2}^{\varphi}+2 \int_{\Omega \times\left\{t_{0}-1\right\}} u \nabla u \cdot \nabla \varphi^{2} G_{\left(x_{0}, t_{0}\right)} \mathrm{d} x
$$

This scales

$$
R \frac{\mathrm{d}}{\mathrm{d} R} F_{2}^{\varphi}(R)=4 D^{\varphi}(R)-2 p F_{p}^{\varphi}(R)+\frac{4}{p-2} F_{2}^{\varphi}(R)+A_{2}^{\varphi}(R)
$$

and may be combined with (3.11) to give

$$
\begin{aligned}
\left.\frac{\mathrm{d}}{\mathrm{d} R} F_{p}^{\varphi}(R)\right|_{R=1}= & \frac{2}{p} \frac{\mathrm{d}}{\mathrm{d} R} D^{\varphi}(R)-\frac{1}{2 p} \frac{\mathrm{d}}{\mathrm{d} R}\left(R \frac{\mathrm{d}}{\mathrm{d} R} F_{2}^{\varphi}(R)-A_{2}^{\varphi}(R)\right)+\frac{2}{p(p-2)} \frac{\mathrm{d}}{\mathrm{d} R} F_{2}^{\varphi}(R) \\
= & \frac{1}{p} \int_{\Omega \times\left\{t_{0}-1\right\}}\left(x \cdot \nabla u-2 u_{t}+\frac{2}{p-2} u\right)^{2} \varphi^{2} G_{\left(X_{0}, t_{0}\right)} \mathrm{d} x+\frac{2}{p}\left(B^{\varphi}+A_{D}^{\varphi}\right) \\
& +\left.\frac{2}{p} \frac{\mathrm{d}}{\mathrm{d} R} F_{p}^{\varphi}(R)\right|_{R=1}-\left.\frac{1}{2 p} \frac{\mathrm{d}}{\mathrm{d} R}\left(R \frac{\mathrm{d}}{\mathrm{d} R} F_{2}^{\varphi}(R)-A_{2}^{\varphi}(R)\right)\right|_{R=1} .
\end{aligned}
$$

Collecting terms, we conclude the following result.

Proposition 3.1. Let $u$ be a smooth solution of (1.2) and let $\varphi$ be a cutoff function as above. Then there holds

$$
\begin{aligned}
& \left.\frac{\mathrm{d}}{\mathrm{d} R}\left(\frac{p-2}{p} F_{p}^{\varphi}(R)+\frac{1}{2 p}\left(R \frac{\mathrm{d}}{\mathrm{d} R} F_{2}^{\varphi}(R)-A_{2}^{\varphi}(R)\right)\right)\right|_{R=1} \\
& \quad=\frac{1}{p} \int_{\Omega \times\left\{t_{0}-1\right\}}\left(x \cdot \nabla u-2 u_{t}+\frac{2}{p-2} u\right)^{2} \varphi^{2} G_{\left(x_{0}, t_{0}\right)} \mathrm{d} x+\frac{2}{p}\left(A_{D}^{\varphi}+B^{\varphi}\right),
\end{aligned}
$$

where $A_{2}^{\varphi}$ is defined in (3.13), and with $A_{D}^{\varphi}, B^{\varphi}$ as defined in (3.12), (3.8), respectively. 
Remark 3.2. (i) For our later purposes it will be convenient to write the term

$$
\begin{aligned}
H^{\varphi}(R) & :=\frac{p-2}{p} F_{p}^{\varphi}(R)+\frac{1}{2 p}\left(R \frac{\mathrm{d}}{\mathrm{d} R} F_{2}^{\varphi}(R)-A_{2}^{\varphi}(R)\right) \\
& =\frac{p-2}{p} F_{p}^{\varphi}(R)+\frac{1}{2 p}\left(\frac{\mathrm{d}}{\mathrm{d} R}\left(R F_{2}^{\varphi}(R)\right)-F_{2}^{\varphi}(R)-A_{2}^{\varphi}(R)\right)
\end{aligned}
$$

appearing on the left of (3.14) in a somewhat different manner. Comparing (3.14) with the Giga-Kohn formula (3.11), we also have

$$
H^{\varphi}(R)=\frac{2}{p} D^{\varphi}(R)-\frac{2}{p} F_{p}^{\varphi}(R)+\frac{2}{p(p-2)} F_{2}^{\varphi}(R) .
$$

We can therefore eliminate $F_{2}(R)$ from the above expressions in a manner similar to Pacard [28] to obtain

$$
H^{\varphi}(R)=\frac{2(p-2)}{p(p+2)}\left(D^{\varphi}(R)+F_{p}^{\varphi}(R)\right)+\frac{2}{p(p+2)}\left(\frac{\mathrm{d}}{\mathrm{d} R}\left(R F_{2}^{\varphi}(R)\right)-A_{2}^{\varphi}(R)\right)
$$

as an equivalent expression for $H^{\varphi}$, which will be very useful later. We also use the notation

$$
H^{\varphi}(R)=H_{u,\left(x_{0}, t_{0}\right)}^{\varphi}(R)=H_{u}^{\varphi}(R)=H_{\left(x_{0}, t_{0}\right)}^{\varphi}(R)
$$

whenever the solution $u$ and/or the center $\left(x_{0}, t_{0}\right)$ of scaling are not clear from the context, and similarly for $D^{\varphi}$, etc.

(ii) For any smooth, bounded $\Omega \subset \mathbb{R}^{n}$ we can find numbers $C_{\Omega}>0, \rho_{0}>0$ such that, for any $0<\rho<\rho_{0}$ and any choice of origin $x_{0}=0 \in \Omega$ with $\operatorname{dist}\left(x_{0}, \partial \Omega\right) \geq C_{\Omega} \rho^{2}$, there holds $x \cdot v \geq 0$ on $B_{2 \rho}(0) \cap \partial \Omega$. We may take $C_{\Omega}=0$ if $\Omega$ is convex. Provided that our cutoff function $\varphi$ is supported in $B_{2 \rho}(0)$, then also $B^{\varphi} \geq 0$ in (3.14). Also estimating the second term in $L_{D}^{\varphi}$ via Young's inequality

$$
\begin{aligned}
& 8 \int_{\Omega \times\left\{t_{0}-1\right\}} \nabla u \cdot \nabla \varphi\left(x \cdot \nabla u-2 u_{t}+\frac{2}{p-2} u\right) \varphi G_{\left(x_{0}, t_{0}\right)} \mathrm{d} x \\
& \leq \int_{\Omega \times\left\{t_{0}-1\right\}}\left(x \cdot \nabla u-2 u_{t}+\frac{2}{p-2} u\right)^{2} \varphi^{2} G_{\left(x_{0}, t_{0}\right)} \mathrm{d} x \\
& \quad+16 \int_{\Omega \times\left\{t_{0}-1\right\}}|\nabla u|^{2}|\nabla \varphi|^{2} G_{\left(x_{0}, t_{0}\right)} \mathrm{d} x
\end{aligned}
$$

from (3.14) we then obtain the bound

$$
\left.\frac{\mathrm{d}}{\mathrm{d} R} H^{\varphi}(R)\right|_{R=1} \geq \frac{1}{2 p} \int_{\Omega \times\left\{t_{0}-1\right\}}\left(x \cdot \nabla u-2 u_{t}+\frac{2}{p-2} u\right)^{2} \varphi^{2} G_{\left(X_{0}, t_{0}\right)} \mathrm{d} x+\frac{2}{p} A_{0}^{\varphi} .
$$


with error term

$$
\begin{aligned}
A_{0}^{\varphi}= & \int_{\Omega \times\left\{t_{0}-1\right\}}|\nabla u|^{2} \varphi x \cdot \nabla \varphi G_{\left(x_{0}, t_{0}\right)} \mathrm{d} x \\
& -4 \int_{\Omega \times\left\{t_{0}-1\right\}}|\nabla u|^{2}|\nabla \varphi|^{2} G_{\left(x_{0}, t_{0}\right)} \mathrm{d} x-L_{p}^{\varphi}+\frac{1}{p-2} L_{2}^{\varphi} \\
= & \int_{\Omega \times\left\{t_{0}-1\right\}}\left(|\nabla u|^{2}-\frac{2}{p}|u|^{p}+\frac{1}{p-2}|u|^{2}\right) \varphi x \cdot \nabla \varphi G_{\left(x_{0}, t_{0}\right)} \mathrm{d} x \\
& -4 \int_{\Omega \times\left\{t_{0}-1\right\}}|\nabla u|^{2}|\nabla \varphi|^{2} G_{\left(x_{0}, t_{0}\right)} \mathrm{d} x .
\end{aligned}
$$

(iii) Still with the choice $x_{0}=0$, for general $R>0$ formula (3.17) reads

$$
R \frac{\mathrm{d}}{\mathrm{d} R} H^{\varphi}(R) \geq \frac{R^{\mu}}{2 p} \int_{\Omega \times\left\{t_{0}-R^{2}\right\}} \frac{\left|x \cdot \nabla u+2\left(t-t_{0}\right) u_{t}+a u\right|^{2}}{\left|t_{0}-t\right|} \varphi^{2} G_{\left(x_{0}, t_{0}\right)} \mathrm{d} x+\frac{2}{p} A_{0}^{\varphi}(R),
$$

where $a=\frac{2}{p-2}$ and

$$
\begin{aligned}
A_{0}^{\varphi}(R)= & R^{\mu} \int_{\Omega \times\left\{t_{0}-R^{2}\right\}}\left(|\nabla u|^{2}-\frac{2}{p}|u|^{p}+R^{-2} \frac{|u|^{2}}{p-2}\right) \varphi x \cdot \nabla \varphi G_{\left(x_{0}, t_{0}\right)} \mathrm{d} x \\
& -4 R^{\mu+2} \int_{\Omega \times\left\{t_{0}-R^{2}\right\}}|\nabla u|^{2}|\nabla \varphi|^{2} G_{\left(x_{0}, t_{0}\right)} \mathrm{d} x .
\end{aligned}
$$

Moreover, we have

$$
\begin{aligned}
A_{2}^{\varphi}(R)= & \frac{R^{\mu-2}}{2} \int_{\Omega \times\left\{t_{0}-R^{2}\right\}}|u|^{2} x \cdot \nabla \varphi^{2} G_{\left(x_{0}, t_{0}\right)} \mathrm{d} x \\
& +2 R^{\mu} \int_{\Omega \times\left\{t_{0}-R^{2}\right\}} u \nabla u \cdot \nabla \varphi^{2} G_{\left(x_{0}, t_{0}\right)} \mathrm{d} x .
\end{aligned}
$$

(iv) Letting $\varphi \equiv 1$, from Proposition 3.1 we obtain the identity

$$
\begin{aligned}
& \left.\frac{\mathrm{d}}{\mathrm{d} R}\left(\frac{p-2}{p} F_{p}(R)+\frac{1}{2 p} R \frac{\mathrm{d}}{\mathrm{d} R} F_{2}(R)\right)\right|_{R=1} \\
& \quad=\frac{1}{p} \int_{\Omega \times\left\{t_{0}-1\right\}}\left(x \cdot \nabla u-2 u_{t}+\frac{2}{p-2} u\right)^{2} G_{\left(x_{0}, t_{0}\right)} \mathrm{d} x+\frac{2}{p} B
\end{aligned}
$$

where $F_{p}=F_{p}^{1}$, etc., and where $B=B^{1}$ is the boundary term

$$
B^{1}=\frac{1}{2} \int_{\partial \Omega \times\left\{t_{0}-1\right\}} v \cdot x|\nabla u|^{2} G_{\left(x_{0}, t_{0}\right)} \mathrm{d} o .
$$


If our domain $\Omega$ is convex, for any choice of origin $x_{0}=0 \in \Omega$ we have $x \cdot v \geq 0$ on all of $\partial \Omega$, and hence $B^{1} \geq 0$ in this case. With the equivalent expression

$$
\begin{aligned}
H(R) & =\frac{p-2}{p} F_{p}(R)+\frac{1}{2 p}\left(\frac{\mathrm{d}}{\mathrm{d} R}\left(R F_{2}(R)\right)-F_{2}(R)\right) \\
& =\frac{2(p-2)}{p(p+2)}\left(D(R)+F_{p}(R)\right)+\frac{2}{p(p+2)} \frac{\mathrm{d}}{\mathrm{d} R}\left(R F_{2}(R)\right),
\end{aligned}
$$

for $H=H^{1}$ we then obtain

$$
\left.\frac{\mathrm{d}}{\mathrm{d} R} H(R)\right|_{R=1} \geq \frac{1}{p} \int_{\Omega \times\left\{t_{0}-1\right\}}\left(x \cdot \nabla u-2 u_{t}+\frac{2}{p-2} u\right)^{2} G_{\left(x_{0}, t_{0}\right)} \mathrm{d} x \geq 0
$$

for any such $x_{0}$ and $t_{0}$.

(v) When $\left(x_{0}, t_{0}\right)=(0,0)$, in the self-similar variables (3.5) we can express

$$
\left(x \cdot \nabla u+2 t u_{t}+\frac{2}{p-2} u\right)(x, t)=-2 \mathrm{e}^{\beta s} w_{s}(y, s) .
$$

\section{The $\varepsilon$-Regularity Theorem}

Given a smoothly bounded domain $\Omega \subset \mathbb{R}^{n}$, for $\left(x_{0}, t_{0}\right) \in \mathbb{R}^{n} \times \mathbb{R}, r>0$ let $Q_{r}\left(x_{0}, t_{0}\right)=$ $P_{r}\left(x_{0}, t_{0}\right) \cap(\Omega \times \mathbb{R})$. Also recall that $\mu=\frac{2 p}{p-2}$.

Proposition 4.1. There are constants $\varepsilon>0$ and $C<\infty$ with the following property: Let $u \in L^{p, \mu}$ be a weak solution to Equation (1.2) on $Q_{1}(0,0)$ and suppose that

$$
\|u\|_{L^{p, \mu}\left(Q_{1}(0,0)\right)} \leq \varepsilon .
$$

Then

$$
\|u\|_{L^{\infty}\left(Q_{1 / 4}(0,0)\right)}+\|\nabla u\|_{L^{\infty}\left(Q_{1 / 8}(0,0)\right)} \leq C\|u\|_{L^{p, \mu}\left(Q_{1}(0,0)\right)} .
$$

For $f \in L^{q}\left(\mathbb{R}^{n+1}\right)$ with compact support we set

$$
S f(x, t):=\int_{-\infty}^{t} \int_{\mathbb{R}^{n}} f(y, s) G(x-y, s-t) \mathrm{d} y \mathrm{~d} s
$$

so that $S f$ solves

$$
(S f)_{t}-\Delta(S f)=f \quad \text { on } \mathbb{R}^{n+1}
$$

By adapting the methods of Adams [1], we can show that $S$ is well-behaved on Morrey spaces. 
Lemma 4.2. For $2<\lambda \leq n+2$ and any $1<q<\frac{\lambda}{2}$ let $s$ be given by $\frac{1}{q}-\frac{1}{s}=\frac{2}{\lambda}$. Then the map

$$
S: L^{q, \lambda}\left(\mathbb{R}^{n+1}\right) \rightarrow L^{s, \lambda}\left(\mathbb{R}^{n+1}\right)
$$

is bounded.

Proof. Similar to Adams [1, proof of Proposition 3.1], we first derive pointwise estimates in terms of the fractional maximal functions

$$
M_{\alpha} f(x, t):=\sup _{r>0} r^{\alpha-(n+2)} \iint_{P_{r}(x, t)}|f(y, s)| \mathrm{d} y \mathrm{~d} s, \quad \alpha>0 .
$$

Note that, from Hölder's inequality, we obtain

$$
\left(M_{\lambda / q} f\right)^{q} \leq M_{\lambda}\left(|f|^{q}\right) \leq\|f\|_{L^{q, \lambda}}^{q} .
$$

For $f \neq 0$ we use the well-known heat kernel estimate

$$
|G(x, t)| \leq C\left(|x|+t^{1 / 2}\right)^{-n}
$$

and, for $\delta>0$ to be chosen later, we split

$$
\begin{aligned}
|S f(x, t)| \leq & C \int_{-\infty}^{t} \int_{\mathbb{R}^{n}}\left(|x-y|+|t-s|^{\frac{1}{2}}\right)^{-n}|f(y, s)| \mathrm{d} y \mathrm{~d} s \\
\leq & C \sum_{k=1}^{\infty} \iint_{P_{\delta 2^{-k+1}}(x, t)-P_{\delta 2^{-k}(x, t)}}\left(|x-y|+|t-s|^{\frac{1}{2}}\right)^{-n}|f(y, s)| \mathrm{d} y \mathrm{~d} s \\
& +C \sum_{k=0}^{\infty} \iint_{P_{\delta 2^{k+1}}(x, t)-P_{\delta 2^{k}(x, t)}}\left(|x-y|+|t-s|^{\frac{1}{2}}\right)^{-n}|f(y, s)| \mathrm{d} y \mathrm{~d} s
\end{aligned}
$$

to obtain the bound

$$
\begin{aligned}
|S f(x, t)| \leq & C \sum_{k=1}^{\infty}\left(\delta 2^{-k}\right)^{-n}\left(\delta 2^{-k+1}\right)^{n+2} M_{0} f(x, t) \\
& +C \sum_{k=0}^{\infty}\left(\delta 2^{k}\right)^{-n}\left(\delta 2^{k+1}\right)^{n+2-\frac{\lambda}{q}} M_{\frac{\lambda}{q}} f(x, t) \\
\leq & C \delta^{2} M_{0} f(x, t)+C \delta^{2-\frac{\lambda}{q}} M_{\frac{\lambda}{q}} f(x, t) .
\end{aligned}
$$


Choosing $\delta=\delta(x, t)=\left(M_{\lambda / q} f(x, t) / M_{0} f(x, t)\right)^{\frac{q}{\lambda}}$, we find

$$
|S f(x, t)| \leq C\left(M_{\lambda / q} f(x, t)\right)^{\frac{2 q}{\lambda}}\left(M_{0} f(x, t)\right)^{1-\frac{2 q}{\lambda}} .
$$

As the space-time $\mathbb{R}^{n+1}$ equiped with the metric

$$
d_{\text {par }}((x, t),(y, s))=\max \left\{|x-y|+|t-s|^{1 / 2}\right\}
$$

and the Lebesgue measure is a doubling measure space, the maximal function $M_{0}$ satisfies an $L^{p}$-estimate for $1<p<\infty$; see [33, Theorem 1, Chapter 1]. Hölder's inequality together with these $L^{p}$ estimates for our choice of $s$ with $1-\frac{2 q}{\lambda}=\frac{q}{s}$ then leads to

$$
\|S f\|_{L^{s}\left(\mathbb{R}^{n+1}\right)} \leq C\left\|M_{\lambda / q} f\right\|_{L^{\infty}\left(\mathbb{R}^{n+1}\right)}^{\frac{2 q}{\lambda}}\|f\|_{L^{q}\left(\mathbb{R}^{n+1}\right)}^{1-\frac{2 q}{\lambda}}
$$

Finally, for $\left(x_{0}, t_{0}\right) \in \mathbb{R}^{n+1}$ we decompose $f=f^{\prime}+f^{\prime \prime}$, where $f^{\prime}=f \chi_{P_{2 r}\left(x_{0}, t_{0}\right)}$. By (4.1), (4.2) then there holds

$$
\left\|S f^{\prime}\right\|_{L^{s}\left(P_{r}\left(x_{0}, t_{0}\right)\right)} \leq C\left\|M_{\lambda / q} f^{\prime}\right\|_{L^{\infty}}^{\frac{2 q}{\lambda}}\left\|f^{\prime}\right\|_{L^{q}}^{1-\frac{2 q}{\lambda}} \leq C r^{\frac{n+2-\lambda}{s}}\|f\|_{L^{q, \lambda}} .
$$

Furthermore, for $(x, t) \in P_{r}\left(x_{0}, t_{0}\right)$ we have

$$
\begin{aligned}
\left|S f^{\prime \prime}(x, t)\right| & \leq C \int_{r}^{\infty} \rho^{-n-1}\left(\int_{P_{\rho}(x, t)}|f(y, s)| \mathrm{d} y \mathrm{~d} s\right) \mathrm{d} \rho \\
& \leq C M_{\lambda / q} f(x, t) \int_{r}^{\infty} \rho^{1-\frac{\lambda}{q}} \mathrm{~d} \rho \leq C r^{2-\frac{\lambda}{q}}\|f\|_{L^{q, \lambda}}=C r^{-\frac{\lambda}{s}}\|f\|_{L^{q, \lambda}}
\end{aligned}
$$

hence

$$
\left\|S f^{\prime \prime}\right\|_{L^{s}\left(P_{r}\left(x_{0}, t_{0}\right)\right)} \leq C r^{\frac{n+2-\lambda}{s}}\|f\|_{L^{q, \lambda}}
$$

which concludes the proof.

Remark 4.3. For a smoothly bounded domain $\Omega \subset \mathbb{R}^{n}$ we let $\Gamma$ be the fundamental solution of the heat equation on $\Omega$. By the maximum principle we then have $0 \leq \Gamma \leq G$; the analog of Lemma 4.2 therefore also holds on any domain.

Proof of Proposition 4.1. We may assume $\epsilon \leq 1$. First suppose that $B_{1}(0) \subset \Omega$. Throughout the following we will use the abbreviation $P_{r}:=P_{r}(0,0)$ for $r>0$. Decompose $u=$ 
$v+w$, where

$$
w=S\left(|u|^{p-2} u \chi_{P_{1}}\right)
$$

Note that $|u|^{p-1} \chi_{P_{1}} \in L^{\frac{p}{p-1}, \mu}$. Observing that $\frac{p-1}{p}-\frac{1}{p}=\frac{2}{\mu}$ from Lemma 4.2 we conclude

$$
\|w\|_{L^{p, \mu}\left(P_{1}\right)} \leq C\left\||u|^{p-1}\right\|_{L^{\frac{p}{p-1}, \mu}\left(P_{1}\right)}=C\|u\|_{L^{p, \mu}\left(P_{1}\right)}^{p-1} \leq C \epsilon^{p-2}\|u\|_{L^{p, \mu}\left(P_{1}\right)}
$$

Since

$$
v_{t}-\Delta v=0 \quad \text { on } P_{1}
$$

and

$$
\|v\|_{L^{p}\left(P_{1}\right)} \leq\|u\|_{L^{p}\left(P_{1}\right)}+\|w\|_{L^{p}\left(P_{1}\right)} \leq C\|u\|_{L^{p, \mu}\left(P_{1}\right)}
$$

parabolic regularity leads to the bound

$$
\|v\|_{L^{\infty}\left(P_{1 / 2}\right)} \leq C\|u\|_{L^{p, \mu}\left(P_{1}\right)} .
$$

Using Hölder's inequality, we hence derive

$$
\|v\|_{L^{p, \mu}\left(P_{\sigma}\right)} \leq C \sigma^{\mu / p}\|u\|_{L^{p, \mu}\left(P_{1}\right)}
$$

for all $\sigma \leq \frac{1}{2}$.

Therefore, if we choose $\sigma, \varepsilon>0$ small enough, we obtain

$$
\begin{aligned}
\|u\|_{L^{p, \mu}\left(P_{\sigma}\right)} & \leq\|w\|_{L^{p, \mu}\left(P_{\sigma}\right)}+\|v\|_{L^{p, \mu}\left(P_{\sigma}\right)} \leq C\left(\varepsilon^{p-2}+\sigma^{\mu / p}\right)\|u\|_{L^{p, \mu}\left(P_{1}\right)} \\
& \leq \frac{1}{2}\|u\|_{L^{p, \mu}\left(P_{1}\right)}
\end{aligned}
$$

Applying this for $\left(x_{0}, t_{0}\right) \in P_{1 / 2}$ and $0<R<\frac{1}{2}$ for the scaled solution $u_{R}(x, t):=$ $R^{2 /(p-2)} u\left(x_{0}+R x, t_{0}+R^{2} t\right)$ which solves (1.2) on $P_{1}$, and using the invariance of $\|\cdot\|_{L^{p, \mu}}$ under this scaling, we find that

$$
\|u\|_{L^{p, \mu}\left(P_{\sigma r}\left(x_{0}, t_{0}\right)\right)} \leq \frac{1}{2}\|u\|_{L^{p, \mu}\left(P_{r}\left(x_{0}, t_{0}\right)\right)}
$$

for all $\left(x_{0}, t_{0}\right) \in P_{1 / 2}$ and $r<\frac{1}{2}$. 
Iterating this inequality proves that, for $\theta>0$ small enough, we have

$$
\|u\|_{L^{p, \mu-\theta}\left(P_{1 / 2}\right)} \leq C\|u\|_{L^{p, \mu}\left(P_{1}\right)}
$$

where $C$ and $\theta$ do not depend on $u$. Clearly, we may assume that $\frac{p}{p-1}<\frac{\mu-\theta}{2}$, and we may decrease $\theta$ further, if necessary.

For any $q \geq p$ with $\frac{q}{p-1}<\frac{\mu-\theta}{2}$ let $s>1$ be given by

$$
\frac{1}{s}=\frac{p-1}{q}-\frac{2}{\mu-\theta} .
$$

Note that

$$
\frac{1}{q}-\frac{1}{s}=\frac{2}{\mu-\theta}-\frac{p-2}{q}=\frac{2}{\mu}\left(\frac{\mu}{\mu-\theta}-\frac{p}{q}\right) \geq \frac{2}{\mu}\left(\frac{\mu}{\mu-\theta}-1\right)=\frac{2 \theta}{\mu(\mu-\theta)}=: \gamma
$$

with a constant $\gamma>0$, so that

$$
s \geq q /(1-q \gamma) \geq q /(1-p \gamma) .
$$

Let $0<r<R<\frac{1}{2}$ and assume that $u \in L^{q, \mu-\theta}\left(P_{R}\right)$ for some $q \geq p$ with $\|u\|_{L^{q, \mu-\theta}\left(P_{R}\right)} \leq$ $C\|u\|_{L^{p, \mu}\left(P_{1}\right)} \leq C$. Suppose that $\frac{q}{p-1}<\frac{\mu-\theta}{2}$ and let $s=s(q)>1$ be given as above. As before, we decompose $u=v+w$ where now

$$
w:=S\left(|u|^{p-2} u \chi_{P_{R}}\right)
$$

Lemma 4.2 now gives the bound

$$
\|w\|_{L^{s, \mu-\theta}\left(P_{R}\right)} \leq C\|u\|_{L^{q, \mu-\theta}\left(P_{R}\right)}^{p-1} .
$$

Again we may use parabolic regularity to see that

$$
\|v\|_{L^{s, \mu-\theta}\left(P_{r}\right)} \leq C\|v\|_{L^{\infty}\left(P_{r}\right)} \leq C\|u\|_{L^{q, \mu-\theta}\left(P_{R}\right)}
$$

for some $C>0$ independent of $u$. Thus, from (4.6) we obtain

$$
\begin{aligned}
\|u\|_{L^{s, \mu-\theta}\left(P_{r}\right)} & \leq C\left(1+\|u\|_{L^{q, \mu-\theta}\left(P_{R}\right)}^{p-2}\right)\|u\|_{L^{q, \mu-\theta}\left(P_{R}\right)} \\
& \leq C\|u\|_{L^{q, \mu-\theta}\left(P_{R}\right)} \leq C\|u\|_{L^{p, \mu}\left(P_{1}\right)} .
\end{aligned}
$$

Choosing $q_{0}=p, R_{0}=\frac{1}{2}$ and letting $q_{k+1}=s\left(q_{k}\right), R_{k}=\frac{1}{4}+2^{-k-2}, r_{k}=R_{k+1}$ for $k \in \mathbb{N}_{0} ，$ as long as $\frac{q_{k}}{p-1}<\frac{\mu-\theta}{2}$, we obtain $u \in L^{q_{k+1}, \mu-\theta}\left(P_{R_{k+1}}\right)$ for each $k=0,1, \ldots$ with a uniform 
bound

$$
\|u\|_{L^{q_{k+1}, \mu-\theta}\left(P_{R_{k+1}}\right)} \leq C\|u\|_{L^{p, \mu}\left(P_{1}\right)}
$$

where $C=C_{k}$ is independent of $u$.

For some number $k_{0} \in \mathbb{N}$ independent of $u$ then we have

$$
\frac{q_{k_{0}-1}}{p-1}<\frac{\mu-\theta}{2} \leq \frac{q_{k_{0}}}{p-1} .
$$

Hence, by Hölder's inequality, also using the fact that $P_{R_{k}} \subset P_{1}$ for each $k$, for any $q_{k_{0}-1} \leq$ $q \leq q_{k_{0}}$ we have

$$
u \in L^{q_{k_{0}}, \mu-\theta}\left(P_{R_{k_{0}}}\right) \subset L^{q, \frac{q(\mu-\theta)}{q k_{0}}}\left(P_{R_{k_{0}}}\right) \subset L^{q, \mu-\theta}\left(P_{R_{k_{0}}}\right)
$$

with

$$
\|u\|_{L^{q, \mu-\theta}\left(P_{R_{k_{0}}}\right)} \leq C\|u\|_{L^{p, \mu}\left(P_{1}\right)}
$$

for some constant $C$ independent of $u$. Letting $\bar{R}:=R_{k_{0}+1}$ and choosing a number $q \in$ $\left[q_{k_{0}-1}, q_{k_{0}}[\right.$ with

$$
\frac{2}{\mu-\theta}<\frac{p-1}{q}<\frac{2}{\mu-\theta}+\frac{2}{(n+2)(p-1)},
$$

there results $u \in L^{s, \mu-\theta}\left(P_{\bar{R}}\right)$ for some $s=s(q)>\frac{(n+2)(p-1)}{2}$, and

$$
\|u\|_{L^{s, \mu-\theta}\left(P_{\bar{R}}\right)} \leq C\|u\|_{L^{p, \mu}\left(P_{1}\right)}
$$

Parabolic regularity then gives

$$
w:=S\left(|u|^{p-2} u \chi_{P_{\bar{R}}}\right) \in L^{\infty}\left(P_{1}\right)
$$

with

$$
\|w\|_{L^{\infty}\left(P_{1}\right)} \leq C\|u\|_{L^{s, \mu-\theta}\left(P_{\bar{R}}\right)} \leq C\|u\|_{L^{p, \mu}\left(P_{1}\right)},
$$

and the asserted bound for $\|u\|_{L^{\infty}\left(P_{1 / 4}\right)}$ follows upon splitting $u=v+w$ with $v$ bounded as in (4.3). The corresponding bound for $|\nabla u|$ now is a consequence of parabolic regularity. In the case when $B_{1}(0) \backslash \Omega \neq \emptyset$ we argue similarly, using Remark 4.3.

\section{The Time-Independent Case}

\subsection{Partial regularity}

By applying Proposition 4.1 to a time-independent weak solution $u \in H^{1} \cap L^{p, \mu}$ of Equation (1.1), we obtain the following result. 
For $x_{0} \in \mathbb{R}^{n}, r>0$ let $\Omega_{r}\left(x_{0}\right)=B_{r}\left(x_{0}\right) \cap \Omega$.

Proposition 5.1. There are constants $\varepsilon>0$ and $C<\infty$ with the following property: If $u \in H^{1} \cap L^{p, \mu}$ is a weak solution to the Equation (1.1) on $\Omega_{1}(0)$ satisfying

$$
\|u\|_{L^{p, \mu}\left(\Omega_{1}(0)\right)} \leq \varepsilon,
$$

then $u$ is smooth in $\Omega_{1 / 4}(0)$ and

$$
\|u\|_{L^{\infty}\left(\Omega_{1 / 4}(0)\right)}+\|\nabla u\|_{L^{\infty}\left(\Omega_{1 / 8}(0)\right)} \leq C\|u\|_{L^{p, \mu}\left(\Omega_{1}(0)\right)} .
$$

For exponents $p \geq 2^{+}=\frac{2(n-1)}{n-3}$ Proposition 5.1 can serve as a substitute for Pacard's [28, Proposition 1], which relies on [28, Lemma 5], where the bound $p<2^{+}$is imposed, and thus allows one to extend the results of Pacard [28] to the full range of exponents $p>2^{*}$. Note that in Proposition 5.1, in contrast to Pacard, we need not assume that $u$ is stationary; it suffices to assume that $u$ is small in the natural Morrey norm.

Adams' [1] potential theoretic methods also offer a direct approach to the proof of Proposition 5.1. Without going into details, we quickly sketch the main ideas. For $f \in L^{q}\left(\mathbb{R}^{n}\right)$ with compact support set

$$
S_{0} f(x):=\int_{\mathbb{R}^{n}} f(y) G_{0}(x-y) \mathrm{d} y,
$$

where $G_{0}(x)=C(n)|x|^{2-n}$ is the Green's function for the Laplacian on $\mathbb{R}^{n}$, so that

$$
-\Delta\left(S_{0} f\right)=f \quad \text { on } \mathbb{R}^{n}
$$

Then, similar to Adams [1, Theorem 3.1] or Lemma 4.2, we have the following result. (The details of the proof can be carried over almost literally.)

Lemma 5.2. For $2<\lambda \leq n$ and any $1<q<\frac{\lambda}{2}$ let $s$ be given by $\frac{1}{q}-\frac{1}{s}=\frac{2}{\lambda}$. Then the map

$$
S_{0}: L^{q, \lambda}\left(\mathbb{R}^{n}\right) \rightarrow L^{s, \lambda}\left(\mathbb{R}^{n}\right)
$$

is bounded.

Remark 5.3. By the maximum principle, we have $0 \leq \Gamma_{0} \leq G_{0}$ for the Green's function $\Gamma_{0}$ of the Laplacian on a domain $\Omega \subset \mathbb{R}^{n}$. Hence the analog of Lemma 5.2 also holds on any domain. 
The proof of Proposition 5.1 then can be completed in the same way as the proof of Proposition 4.1.

\subsection{Morrey space embedding}

As a second side remark we note the following results similar to (a special case of) Adams' [1, Theorem 3.2].

Proposition 5.4. For $1<\lambda \leq n$ and any $1<q<\lambda$ let $s$ be given by $\frac{1}{q}-\frac{1}{s}=\frac{1}{\lambda}$. There is a constant $C<\infty$ with the following properties: If $u \in W_{0}^{1, q}(\Omega)$ satisfies $\nabla u \in L^{q, \lambda}(\Omega)$, then $u \in L^{s, \lambda}(\Omega)$ and $\|u\|_{L^{s, \lambda}(\Omega)} \leq C\|\nabla u\|_{L^{q, \lambda}(\Omega)}$.

Proof. Extend $u \equiv 0$ outside $\Omega$. Using the representation

$$
u(x)=\int_{\Omega} \nabla u(y) \nabla G_{0}(x-y) \mathrm{d} y,
$$

and observing that $\left|\nabla G_{0}(x-y)\right| \leq C|x-y|^{1-n}$, similar to [1, proof of Proposition 3.1] or the proof of Lemma 4.2 for any $x \in \Omega$ we can bound

$$
|u(x)| \leq C\left(M_{\lambda / q}|\nabla u|(x)\right)^{\frac{q}{\lambda}}\left(M_{0}|\nabla u|(x)\right)^{1-\frac{q}{\lambda}} .
$$

From Hölder's inequality and the $L^{q}$-estimate for the maximal function in view of the identity $1-\frac{q}{\lambda}=\frac{q}{s}$, then we obtain

$$
\|u\|_{L^{s}(\Omega)} \leq C\left\|M_{\lambda / q}|\nabla u|\right\|_{L^{\infty}(\Omega)}^{\frac{q}{\lambda}}\|\nabla u\|_{L^{q}(\Omega)}^{1-\frac{q}{\lambda}} .
$$

For any $x_{0} \in \mathbb{R}^{n}$ and any $r>0$ upon decomposing $f:=|\nabla u|=f^{\prime}+f^{\prime \prime}$, where $f^{\prime}=f \chi_{B_{2 r}\left(x_{0}\right)}$, and letting $u=u^{\prime}+u^{\prime \prime}$ from the above estimate then we obtain

$$
\left\|u^{\prime}\right\|_{L^{s}\left(B_{r}\left(x_{0}\right)\right)} \leq C\left\|M_{\lambda / q} f^{\prime}\right\|_{L^{\infty}}^{\frac{q}{\lambda}}\left\|f^{\prime}\right\|_{L^{q}\left(B_{2 r}\left(x_{0}\right)\right)}^{1-\frac{q}{\lambda}} \leq C r^{\frac{n-\lambda}{s}}\|\nabla u\|_{L^{q, \lambda}(\Omega)} .
$$

Furthermore, for $x \in B_{r}\left(x_{0}\right)$ we have

$$
\begin{aligned}
\left|u^{\prime \prime}(x)\right| & \leq C \int_{r}^{\infty} \rho^{-n}\left(\int_{B_{\rho}\left(x_{0}\right)}|\nabla u(y)| \mathrm{d} y\right) \mathrm{d} \rho \\
& \leq C M_{\lambda / q}|\nabla u|(x) \int_{r}^{\infty} \rho^{-\frac{\lambda}{q}} \mathrm{~d} \rho \leq C r^{1-\frac{\lambda}{q}}\|\nabla u\|_{L^{q, \lambda}(\Omega)}=C r^{-\frac{\lambda}{s}}\|\nabla u\|_{L^{q, \lambda}(\Omega)}
\end{aligned}
$$


and hence

$$
\left\|u^{\prime \prime}\right\|_{L^{s}\left(B_{r}\left(X_{0}\right)\right)} \leq C r^{\frac{n-\lambda}{s}}\|\nabla u\|_{L^{q, \lambda}(\Omega)},
$$

which concludes the proof.

Similarly, we obtain derivative estimates. Again let $\Gamma_{0}$ be the Green's function on $\Omega$. Note the following simple consequence of the maximum principle. A proof can be found in [12, Theorem 4.7, p. 105].

Lemma 5.5. There is $C>0$ such that, for any $x, y \in \Omega$, we have

$$
\left|\nabla \Gamma_{0}(x, y)\right| \leq C|x-y|^{1-n} .
$$

We can use this bound to obtain the following result.

Proposition 5.6. For $1<\lambda \leq n$ and any $1<q<\lambda$ let $s$ be given by $\frac{1}{q}-\frac{1}{s}=\frac{1}{\lambda}$. There is a constant $C<\infty$ with the following properties: If $u \in W^{2, q} \cap W_{0}^{1, q}(\Omega)$ satisfies $\Delta u \in L^{q, \lambda}(\Omega)$, then $\nabla u \in L^{s, \lambda}(\Omega)$ and

$$
\|\nabla u\|_{L^{s, \lambda}(\Omega)} \leq C\|\Delta u\|_{L^{q, \lambda}(\Omega)} .
$$

Proof. Extend $\Delta u=0$ on $\mathbb{R}^{n} \backslash \Omega$. Using the representations

$$
u(x)=-\int_{\Omega} \Delta u(y) \Gamma_{0}(x, y) \mathrm{d} y, \quad \nabla u(x)=-\int_{\Omega} \Delta u(y) \nabla \Gamma_{0}(x, y) \mathrm{d} y,
$$

in view of Lemma 5.5 we can bound

$$
|\nabla u(x)| \leq C \int_{\mathbb{R}^{n}}|x-y|^{1-n}|\Delta u(y)| d y .
$$

Again, following [1, proof of Proposition 3.1] then we find the estimate

$$
|\nabla u(x)| \leq C\left(M_{\lambda / q}|\Delta u|(x)\right)^{\frac{q}{\lambda}}\left(M_{0}|\Delta u|(x)\right)^{1-\frac{q}{\lambda}} .
$$

Hölder's inequality together with the $L^{q}$-estimate for the maximal function and the identity $1-\frac{q}{\lambda}=\frac{q}{s}$ then lead to the bound

$$
\|\nabla u\|_{L^{s}(\Omega)} \leq C\left\|M_{\lambda / q} \mid \Delta u\right\|_{L^{\infty}(\Omega)}^{\frac{q}{2}}\|\Delta u\|_{L^{q}(\Omega)}^{1-\frac{q}{2}} .
$$


Given $x_{0} \in \mathbb{R}^{n}$, we decompose $f:=\Delta u=f^{\prime}+f^{\prime \prime}$ where $f^{\prime}=f \chi_{B_{2 r}\left(x_{0}\right)}$, and let $u=u^{\prime}+u^{\prime \prime}$, where

$$
u^{\prime}(x)=\int_{\Omega} f^{\prime}(y) \Gamma_{0}(x, y) \mathrm{d} y
$$

and similarly for $u^{\prime \prime}$. By (5.1) then we have

$$
\left\|\nabla u^{\prime}\right\|_{L^{s}\left(\Omega_{r}\left(x_{0}\right)\right)} \leq C\left\|M_{\lambda / q}\left|f^{\prime}\right|\right\|_{L^{\infty}}^{\frac{q}{\lambda}}\left\|f^{\prime}\right\|_{L^{q}\left(\Omega_{2 r}\left(x_{0}\right)\right)}^{1-\frac{q}{\lambda}} \leq C r^{\frac{n-\lambda}{s}}\|\Delta u\|_{L^{q, \lambda}(\Omega)} .
$$

Furthermore, for $x \in B_{r}\left(x_{0}\right)$ there holds

$$
\begin{aligned}
\left|\nabla u^{\prime \prime}(x)\right| & \leq C \int_{r}^{\infty} \rho^{-n}\left(\int_{B_{\rho}\left(x_{0}\right)}|\Delta u(y)| \mathrm{d} y\right) \mathrm{d} \rho \\
& \leq C M_{\lambda / q}|\Delta u|(x) \int_{r}^{\infty} \rho^{-\frac{\lambda}{q}} \mathrm{~d} \rho \\
& \leq C r^{1-\frac{\lambda}{q}}\|\Delta u\|_{L^{q, \lambda}(\Omega)}=C r^{-\frac{\lambda}{s}}\|\Delta u\|_{L^{q, \lambda}(\Omega)}
\end{aligned}
$$

and hence

$$
\left\|\nabla u^{\prime \prime}\right\|_{L^{s}\left(\Omega_{r}\left(x_{0}\right)\right)} \leq C r^{\frac{n-\lambda}{s}}\|\Delta u\|_{L^{q, \lambda}(\Omega)} .
$$

The proof is complete.

We conclude that there is a threshold for the scaled energy of nontrivial entire solutions to (1.1) on any bounded domain $\Omega \subset \mathbb{R}^{n}$.

Proposition 5.7. There is a constant $\varepsilon>0$ with the following property: Let $u \in H^{1} \cap$ $L^{p, \mu}(\Omega)$ weakly solve (1.1). Then either $u \equiv 0$, or we have $\|u\|_{L^{p, \mu}(\Omega)} \geq \varepsilon$.

Proof. Combining the results of Propositions 5.4 and 5.6, recalling that $\frac{1}{2}=\frac{p-1}{p}-\frac{1}{\mu}$, we find the chain of estimates

$$
\|\nabla u\|_{L^{2, \mu}(\Omega)} \leq C\|\Delta u\|_{L^{\frac{p}{p-1}, \mu}(\Omega)}=C\|u\|_{L^{p, \mu}((\Omega)}^{p-1} \leq C\|\nabla u\|_{L^{2, \mu}(\Omega)}^{p-1} .
$$

The claim follows.

The above results naturally lead to the question if, analogous to the case of Sobolev's embedding $\dot{H}^{1}\left(\mathbb{R}^{n}\right) \hookrightarrow L^{2^{*}}\left(\mathbb{R}^{n}\right)$, the best constant for the Morrey estimate $\|u\|_{L^{p, \mu}\left(\left(\mathbb{R}^{n}\right)\right.} \leq C\|\nabla u\|_{L^{2, \mu}\left(\mathbb{R}^{n}\right)}$ given by Proposition 5.4 is attained at a function $v>0$, and if 
the corresponding $v$ induces a solution of (1.1), which then might be called a "ground state".

\section{Applications}

We now apply the results of the preceding sections to the flow (1.2), (3.1). Given smooth initial data $u_{0}$, there is a smooth solution $u \in C^{\infty}(\Omega \times[0, T[)$ of (1.2), (3.1), defined on a maximal time interval [0, $T$ [ for some $T>0$. Our aim is to show that Proposition 3.1 can be exploited to obtain bounds for $u$ in the natural Morrey spaces so that the results from Section 4 may be applied. We start by deriving estimates for the functions $H$ and/or $H^{\varphi}$ both at the initial time and close to any fixed point $\left(x_{0}, t_{0}\right) \in \Omega \times[0, T[$.

\subsection{Upper bounds for $H$ and $H^{\varphi}$ at $t=0$}

Fix any $x_{0} \in \bar{\Omega}, t_{0}>0$, and let $0<R \leq R_{0}=\sqrt{t_{0}}>0$. We shift coordinates so that $x_{0}=0$, as usual. The expression (3.15) is the most suitable for bounding

$$
\begin{aligned}
H(R) & =H_{\left(x_{0}, t_{0}\right)}^{1}(R)=\frac{p-2}{p} F_{p}(R)+\frac{1}{2 p} R \frac{\mathrm{d}}{\mathrm{d} R} F_{2}(R) \\
& =\frac{2}{p}\left(D(R)-F_{p}(R)\right)+\frac{2}{p(p-2)} F_{2}(R)
\end{aligned}
$$

from above. By Hölder's inequality and in view of the fact that $G_{*}$ defines a probability measure on $\mathbb{R}^{n}$, we can bound

$$
F_{2}(R) \leq F_{p}^{2 / p}(R)
$$

and from (6.1) we obtain the bound

$$
H(R) \leq \frac{2}{p}\left(D-F_{p}+\frac{1}{p-2} F_{p}^{2 / p}\right)(R) .
$$

The functions involved behave well with respect to the Morrey norms considered above. Letting $R_{k}=2{ }^{k} R_{0}, k \in \mathbb{N}$, upon bounding

$$
G_{\left(x_{0}, t_{0}\right)}(x, 0)=C R_{0}^{-n} \mathrm{e}^{-\frac{\left|x-x_{0}\right|^{2}}{4 R_{0}^{2}}} \leq C R_{0}^{-n} \mathrm{e}^{-2^{2(k-2)}} \quad \text { on } B_{R_{k}} \backslash B_{R_{k-1}}\left(x_{0}\right), k \in \mathbb{N} \text {, }
$$


for any initial data (3.1) we find

$$
\begin{aligned}
2 D\left(R_{0}\right) & =R_{0}^{\mu} \int_{\Omega \times\{0\}}\left|\nabla u_{0}\right|^{2} G_{\left(x_{0}, t_{0}\right)} \mathrm{d} x \\
& \leq C \sum_{k=1}^{\infty} 2^{k(n-\mu)} \mathrm{e}^{-2^{2(k-2)}} R_{k}^{\mu-n} \int_{B_{R_{k} \backslash B_{R_{k-1}}\left(x_{0}\right)}\left|\nabla u_{0}\right|^{2} \mathrm{~d} x} \\
& \leq C \sup _{R \geq R_{0}} R^{\mu-n} \int_{B_{R}\left(x_{0}\right)}\left|\nabla u_{0}\right|^{2} \mathrm{~d} x \leq C\left\|\nabla u_{0}\right\|_{L^{2, \mu}}^{2} .
\end{aligned}
$$

Similarly, we can estimate

$$
\begin{aligned}
2 F_{2}\left(R_{0}\right) & =R_{0}^{\mu-2} \int_{\Omega \times\{0\}}\left|u_{0}\right|^{2} G_{\left(x_{0}, t_{0}\right)} \mathrm{d} x \\
& \leq C \sup _{R \geq R_{0}} R^{\mu-2-n} \int_{B_{R}\left(x_{0}\right)}\left|u_{0}\right|^{2} \mathrm{~d} x \leq C\left\|u_{0}\right\|_{L^{2, \mu-2}}^{2} .
\end{aligned}
$$

Using the fact that $2 \mu / p=\mu-2$ and again using also Hölder's inequality

$$
R^{\mu-2-n} \int_{B_{R}\left(x_{0}\right)}\left|u_{0}\right|^{2} \mathrm{~d} x \leq C\left(R^{\mu-n} \int_{B_{R}\left(x_{0}\right)}\left|u_{0}\right|^{p} \mathrm{~d} x\right)^{2 / p}
$$

we can also bound

$$
\left\|u_{0}\right\|_{L^{2, \mu-2}}^{2} \leq C\left\|u_{0}\right\|_{L^{p, \mu}}^{2}
$$

to obtain

$$
H\left(R_{0}\right) \leq C\left\|\nabla u_{0}\right\|_{L^{2, \mu}}^{2}+C\left\|u_{0}\right\|_{L^{2, \mu-2}}^{2} \leq C\left\|\nabla u_{0}\right\|_{L^{2, \mu}}^{2}+C\left\|u_{0}\right\|_{L^{p, \mu}}^{2}
$$

Of course, we can also estimate

$$
\begin{aligned}
H\left(R_{0}\right) & \leq C D\left(R_{0}\right)+C F_{2}\left(R_{0}\right) \\
& \leq C R_{0}^{\mu} \int_{\Omega \times\{0\}}\left|\nabla u_{0}\right|^{2} G_{\left(x_{0}, t_{0}\right)} \mathrm{d} x+C R_{0}^{\mu-2} \int_{\Omega \times\{0\}}\left|u_{0}\right|^{2} G_{\left(x_{0}, t_{0}\right)} \mathrm{d} x \\
& \leq C R_{0}^{\mu}\left\|\nabla u_{0}\right\|_{L^{\infty}}^{2}+C_{0} R_{0}^{\mu-2}\left\|u_{0}\right\|_{L^{\infty}}^{2},
\end{aligned}
$$

or simply bound

$$
H\left(R_{0}\right) \leq C R_{0}^{\mu-n}\left\|\nabla u_{0}\right\|_{L^{2}}^{2}+C_{0} R_{0}^{\mu-2-n}\left\|u_{0}\right\|_{L^{2}}^{2}
$$

The previous estimates rely on global bounds for the data. For bounding $H^{\varphi}$ with a compactly supported cutoff function $\varphi$, only local estimates are needed and we have bounds similar to the ones above. 


\subsection{Lower bounds for $\boldsymbol{H}$ and Morrey estimates}

For the derivation of Morrey estimates we now use the equivalent expressions (3.20) and (3.16) of $H$ and $H^{\varphi}$.

First consider the case when the domain $\Omega$ is convex, and let $u \in C^{\infty}(\bar{\Omega} \times[0, T[)$ be a solution to (1.2), (3.1). Fix some point $x_{1}=0$ and let $0<T / 2<t_{2}<T$. Observe that, for the choice $\left(x_{1}, t_{2}\right)$ as the center of scaling, letting $u_{R}(x, t)=R^{2 /(p-2)} u\left(x_{1}+R x, t_{2}+R^{2} t\right)$, there holds $r F_{2}(r) \rightarrow 0$ as $r \downarrow 0$. Thus, for $0<R<R_{2}=\sqrt{t_{2}}$ by (3.20), (3.21), for $H=H_{\left(X_{1}, t_{2}\right)}$ we have

$$
H(R) \geq R^{-1} \int_{0}^{R} H(r) \mathrm{d} r \geq \frac{2(p-2)}{p(p+2)} R^{-1} \int_{0}^{R}\left(D+F_{p}\right)(r) \mathrm{d} r .
$$

Note that, for any such $t_{2}<T, R<R_{2}=\sqrt{t_{2}}$ with an absolute constant $c_{0}>0$ there holds

$$
G_{\left(X_{1}, t_{2}\right)} \geq c_{0} R^{-n}>0 \quad \text { on } P_{R / 2}\left(X_{1}, t_{2}-R^{2} / 4\right) .
$$

Extending $u$ by $u=0$ outside $\Omega$ and substituting $t=t_{2}-r^{2}$, we thus obtain the estimate

$$
\begin{aligned}
R^{\mu-n-2} \int_{P_{R / 2}\left(x_{1}, t_{2}-R^{2} / 4\right)}\left(|\nabla u|^{2}+|u|^{p}\right) \mathrm{d} x \mathrm{~d} t & \leq C R^{-2} \int_{R / 2}^{R}\left(D(r)+F_{p}(r)\right) r \mathrm{~d} r \\
& \leq C H(R) \leq C H\left(R_{2}\right)=C H_{\left(x_{1}, t_{2}\right)}\left(\sqrt{t_{2}}\right) .
\end{aligned}
$$

But at time $t=0$, for any $0<t_{2}<T$ we can bound

$$
G_{\left(X_{1}, t_{2}\right)}(x, 0)=C t_{2}^{-n / 2} \mathrm{e}^{-\frac{\left|x-x_{1}\right|^{2}}{4 t_{2}}} \leq\left(T / t_{2}\right)^{n / 2} G_{\left(X_{1}, T\right)}(x, 0) .
$$

Hence, for any $0<R^{2}<T / 2<t_{2}<T$ with a uniform constant $C>0$ there holds

$$
R^{\mu-n-2} \int_{P_{R / 2}\left(X_{1}, t_{2}-R^{2} / 4\right)}\left(|\nabla u|^{2}+|u|^{p}\right) \mathrm{d} x \mathrm{~d} t \leq C H_{\left(X_{1}, T\right)}(\sqrt{T}),
$$

where the right-hand side is now independent of $t_{2}$.

Fixing $x_{0} \in \Omega$ and any time $\left.t_{0} \in\right] \frac{7}{8} T, T\left[\right.$, we let $r_{0}=\sqrt{T-t_{0}}$. For any $\left(x_{1}, t_{1}\right) \in$ $\left.P_{r_{0}}\left(x_{0}, t_{0}\right)\right)$ and any $0<R<2 r_{0}$ we set $t_{2}=t_{1}+R^{2} / 4$. Since $R^{2}<4 r_{0}^{2}=4\left(T-t_{0}\right)<T / 2$, we may then use (6.8) and (6.6) to obtain the bound

$$
\begin{aligned}
& \|\nabla u\|_{L^{2, \mu}\left(P_{r_{0}}\left(x_{0}, t_{0}\right)\right)}+\|u\|_{L^{p, \mu}\left(P_{r_{0}}\left(x_{0}, t_{0}\right)\right)} \\
& \quad \leq C \sup _{\left|x_{1}-x_{0}\right|<r_{0}} H_{\left(X_{1}, T\right)}(\sqrt{T}) \leq C T^{\frac{\mu-n}{2}}\left(\left\|\nabla u_{0}\right\|_{L^{2}}^{2}+T^{-1}\left\|u_{0}\right\|_{L^{2}}^{2}\right),
\end{aligned}
$$


or we may use (6.8) and (6.4) to find

$$
\|\nabla u\|_{L^{2, \mu}\left(P_{r_{0}}\left(x_{0}, t_{0}\right)\right)}+\|u\|_{L^{p, \mu}\left(P_{r_{0}}\left(x_{0}, t_{0}\right)\right)} \leq C\left(\left\|\nabla u_{0}\right\|_{L^{2, \mu}}^{2}+\left\|u_{0}\right\|_{L^{p, \mu}}^{2}\right)
$$

that is, up to a uniform constant $C>0$ the flow (1.2) preserves the Morrey bounds of the data, however, on domains that naturally shrink as we approach the blow-up time.

\subsection{Lower bounds for $\mathrm{H}^{\varphi}$}

For an arbitrary smoothly bounded domain $\Omega$, we choose $\rho_{0}>0, C_{\Omega} \geq 0$ as in Remark 3.2 such that, for any $0<\rho<\rho_{0}$, we have $\left(x-x_{0}\right) \cdot v \geq 0$ on $\partial \Omega \cap B_{2 \rho_{0}}\left(x_{0}\right)$ whenever $x_{0} \in \Omega^{\rho}$, where

$$
\Omega^{\rho}:=\left\{x \in \Omega ; \operatorname{dist}(x, \partial \Omega) \geq C_{\Omega} \rho^{2}\right\} .
$$

Recall that if $\Omega$ is convex, we may let $C_{\Omega}=0$ so that $\Omega^{\rho}=\bar{\Omega}$ for any $0<\rho<\rho_{0}$. With no loss of generality, we may assume that $\rho_{0}<1$. Also set

$$
\delta(\rho, R)= \begin{cases}R^{-n} \mathrm{e}^{-\frac{\rho^{2}}{4 R^{2}}} & \text { if } R \leq \rho, \\ \rho^{-n}\left(1+R^{2} \rho^{\mu-2}\right) & \text { else. }\end{cases}
$$

Fix a smooth cutoff function $\eta$ such that $\chi_{B_{1}(0)} \leq \eta \leq \chi_{B_{2}(0)},|\nabla \eta| \leq 4$ and, for any number $0<\rho<\rho_{0}$, let $\varphi(x)=\varphi^{\rho}(x)=\eta(x / \rho)$. For the function $H^{\varphi^{\rho}}$ then we have the following perturbed monotonicity result.

Proposition 6.1. For any smooth solution $u$ of (1.2) on $\Omega \times\left[0, T\right.$, any $0<\rho<\rho_{0}$, any $x_{1} \in \Omega^{\rho}$, and any $0<R \leq R_{1} \leq \sqrt{T}$ there holds

$$
H_{\left(X_{1}, T\right)}^{\varphi^{\rho}}(R) \leq H_{\left(X_{1}, T\right)}^{\varphi^{\rho}}\left(R_{1}\right)+C \delta\left(\rho, R_{1}\right)
$$

Moreover, the following analog of (6.9) holds. Recall that $\Omega_{r}(x)=\Omega \cap B_{r}(x)$.

Proposition 6.2. Let $u$ be a smooth solution of (1.2) on $\Omega \times\left[0, T\left[\right.\right.$ and let $x_{0} \in \Omega$. Then, for any $0<\rho<\rho_{0}$, any $R_{1}, r_{0}>0$ such that $2 r_{0}<\rho, \Omega_{r_{0}}\left(x_{0}\right) \subset \Omega^{\rho}, 8 r_{0}^{2} \leq R_{1}^{2} \leq T$, letting $t_{0}=$ $T-r_{0}^{2}$, we have

$$
\|\nabla u\|_{L^{2, \mu}\left(P_{r_{0}}\left(x_{0}, t_{0}\right)\right)}^{2}+\|u\|_{L^{p, \mu}\left(P_{r_{0}}\left(x_{0}, t_{0}\right)\right)}^{p} \leq C \sup _{\left|x_{1}-x_{0}\right|<r_{0}} H_{\left(X_{1}, T\right)}^{\varphi^{\rho}}\left(R_{1}\right)+C \delta\left(\rho, R_{1}\right)
$$


For the proof of these results we fix $0<\rho<\rho_{0}$ and again write $\varphi$ instead of $\varphi^{\rho}$ for brevity.

For any choice of origin $0=x_{1} \in \Omega^{\rho}$ and any $0<T / 2<t_{2}<T$, with $\left(x_{1}, t_{2}\right)$ as the center of scaling as before we have $r F_{2}^{\varphi}(r) \rightarrow 0$ as $r \downarrow 0$. Proposition 3.1, (3.16), and (3.18) then yield

$$
\begin{aligned}
\frac{1}{R} \int_{0}^{R}\left(D^{\varphi}(r)+F^{\varphi}(r)\right) \mathrm{d} r \leq & \frac{C}{R} \int_{0}^{R}\left(H^{\varphi}(r)+\left|A_{2}^{\varphi}(r)\right|\right) \mathrm{d} r \\
& \leq C H^{\varphi}(R)+\frac{C}{R} \int_{0}^{R}\left|A_{2}^{\varphi}(r)\right| \mathrm{d} r+C \int_{0}^{R}\left|A_{0}^{\varphi}(r)\right| r^{-1} \mathrm{~d} r
\end{aligned}
$$

and

$$
H^{\varphi}(R) \leq H^{\varphi}\left(R_{1}\right)+C \int_{R}^{R_{1}}\left|A_{0}^{\varphi}(r)\right| r^{-1} \mathrm{~d} r
$$

for any $0<R \leq R_{1} \leq \sqrt{t_{2}}$.

In view of the exponential decay of $G_{\left(x_{1}, t_{2}\right)}$ we can easily bound the error terms given by $A_{0}^{\varphi}$ and $A_{2}^{\varphi}$ by means of the following estimate. Observe that this bound agrees with the blow-up rate $v^{p}(t) \sim(T-t)^{-\frac{p}{p-2}}$ of a solution $v=v(t)>0$ to the ordinary differential equation $v_{t}=v^{p-1}$ blowing up at time $T>0$.

Lemma 6.3. Let $u \in C^{\infty}(\Omega \times[0, T[)$ be a smooth solution to (1.2) for smooth initial data $u_{0}$. There exist a uniform constant $C_{1}>0$ and a constant $C>0$ depending only on $E\left(u_{0}\right)$ such that, for any $0<t_{1}<T$, letting $t_{2}=\left(T+t_{1}\right) / 2$ we have the bound

$$
\int_{t_{1}}^{t_{2}} \int_{\Omega}\left(|\nabla u|^{2}+|u|^{p}\right) \mathrm{d} x \mathrm{~d} t \leq C_{1}\left(T-t_{1}\right)^{-\frac{2}{p-2}}+C\left(1+T-t_{1}\right) .
$$

Proof. Multiplying (1.2) by $u$ and integrating by parts, for any $0<t<T$ we obtain

$$
\frac{1}{2} \frac{\mathrm{d}}{\mathrm{d} t}\left(\int_{\Omega}|u(t)|^{2} \mathrm{~d} x\right)+\int_{\Omega \times\{t\}}\left(|\nabla u|^{2}-|u|^{p}\right) \mathrm{d} x=0
$$

Comparing with the expression for $E(u(t))$ and recalling the energy identity (3.2), we find the equation

$$
\frac{1}{2} \frac{\mathrm{d}}{\mathrm{d} t}\left(\int_{\Omega}|u(t)|^{2} \mathrm{~d} x\right)=\frac{p-2}{p} \int_{\Omega}|u|^{p} \mathrm{~d} x-2 E\left(u(t) \geq \frac{p-2}{p} \int_{\Omega}|u|^{p} \mathrm{~d} x-2 E\left(u_{0}\right) .\right.
$$


Hence, by Hölder's inequality for any $0<t_{1}<t<T$ we obtain the bound

$$
\begin{aligned}
C\|u(t)\|_{L^{p}(\Omega)}^{2} & \geq\|u(t)\|_{L^{2}(\Omega)}^{2} \\
& \geq\left\|u\left(t_{1}\right)\right\|_{L^{2}(\Omega)}^{2}+4 \int_{t_{1}}^{t}\left(\frac{p-2}{2 p}\|u(s)\|_{L^{p}(\Omega)}^{p}-E\left(u_{0}\right)\right) \mathrm{d} s .
\end{aligned}
$$

For $t_{1}<t<T$ set

$$
g=g(t)=\int_{t_{1}}^{t}\left(\frac{p-2}{2 p}\|u(s)\|_{L^{p}(\Omega)}^{p}-E\left(u_{0}\right)\right) \mathrm{d} s
$$

Then, from (6.14) with a uniform constant $c_{0}>0$ we find that

$$
\frac{\mathrm{d}}{\mathrm{d} t} g=\frac{p-2}{2 p}\|u(t)\|_{L^{p(\Omega)}}^{p}-E\left(u_{0}\right) \geq 2 c_{0} g^{p / 2}-E\left(u_{0}\right),
$$

and the latter is larger than $c_{0} g^{p / 2}$ for all $t_{0} \leq t<T$, where $t_{0} \in\left[t_{1}, T\right.$ [ is minimal with the property that $c_{0} g^{p / 2}\left(t_{0}\right) \geq E_{+}\left(u_{0}\right)=\max \left\{E\left(u_{0}\right), 0\right\}$. (We let $t_{0}=T$ if $c_{0} g^{p / 2}(t)<E_{+}\left(u_{0}\right)$ for all $t \in\left[t_{1}, T\right.$. $)$ Recalling that $\mu=\frac{2 p}{p-2}$, in the case where $t_{2}=\left(T+t_{1}\right) / 2 \leq t_{0}$ we have

$$
\begin{aligned}
\mu^{-1} \int_{t_{1}}^{t_{2}}\|u(s)\|_{L^{p}(\Omega)}^{p} \mathrm{~d} s & =g\left(t_{2}\right)+\left(t_{2}-t_{1}\right) E\left(u_{0}\right) \\
& \leq\left(E_{+}\left(u_{0}\right) / c_{0}\right)^{2 / p}+\left(T-t_{1}\right) E\left(u_{0}\right) .
\end{aligned}
$$

On the other hand, if $t_{2}>t_{0}$, upon integrating (6.15) from $t_{2}$ to $T$, we obtain

$$
g\left(t_{2}\right) \leq\left(c_{0} \frac{p-2}{2}\left(T-t_{2}\right)\right)^{-\frac{2}{p-2}}=\left(c_{0} \frac{p-2}{4}\left(T-t_{1}\right)\right)^{-\frac{2}{p-2}}
$$

which gives

$$
\mu^{-1} \int_{t_{1}}^{t_{2}}\|u(s)\|_{L^{p}(\Omega)}^{p} \mathrm{~d} s \leq C_{1}\left(T-t_{1}\right)^{-\frac{2}{p-2}}+\left(T-t_{1}\right) E\left(u_{0}\right) .
$$

The corresponding bound for $\|\nabla u(t)\|_{L^{2}(\Omega)}$ follows from combining the bound for $\|u(t)\|_{L^{p}(\Omega)}$ and the uniform energy bound.

Lemma 6.4. For any $0<R \leq \sqrt{t_{2}}$ there holds

$$
\frac{1}{R} \int_{0}^{R}\left|A_{2}^{\varphi}(r)\right| \mathrm{d} r+\int_{0}^{R}\left|A_{0}^{\varphi}(r)\right| r^{-1} \mathrm{~d} r \leq C \delta(\rho, R) .
$$


Proof. Let $\mathcal{A}=\mathcal{A}_{\rho}\left(x_{1}\right):=\Omega \cap\left(B_{2 \rho}\left(x_{1}\right) \backslash B_{\rho}\left(x_{1}\right)\right)$. With the help of Young's inequality $r^{\mu-2}|u|^{2} \leq r^{\mu}|u|^{p}+C$ we can easily bound

$$
\begin{aligned}
A_{0}^{\varphi}(r)= & \int_{\Omega \times\left\{t_{2}-r^{2}\right\}}\left(r^{\mu}|\nabla u|^{2}-\frac{2}{p} r^{\mu}|u|^{p}+r^{\mu-2} \frac{1}{p-2}|u|^{2}\right) \varphi x \cdot \nabla \varphi G_{\left(X_{1}, t_{2}\right)} \mathrm{d} x \\
& -4 \int_{\Omega \times\left\{t_{2}-r^{2}\right\}}\left(r^{\mu+2}|\nabla u|^{2}|\nabla \varphi|^{2} G_{\left(X_{1}, t_{2}\right)} \mathrm{d} x\right. \\
\leq & C r^{\mu}\left(1+r^{2} \rho^{-2}\right) \int_{\mathcal{A} \times\left\{t_{2}-r^{2}\right\}}\left(|\nabla u|^{2}+|u|^{p}\right) G_{\left(X_{1}, t_{2}\right)} \mathrm{d} x \\
& +C \int_{\mathcal{A} \times\left\{t_{2}-r^{2}\right\}} G_{\left(X_{1}, t_{2}\right)} \mathrm{d} x .
\end{aligned}
$$

Similarly, we have

$$
\begin{aligned}
A_{2}^{\varphi}(r)= & \int_{\Omega \times\left\{t_{2}-r^{2}\right\}}\left(r^{\mu-2}|u|^{2} x \cdot \nabla \varphi+4 r^{\mu} u \nabla u \cdot \nabla \varphi\right) \varphi G_{\left(x_{1}, t_{2}\right)} \mathrm{d} x \\
\leq & C \int_{\mathcal{A} \times\left\{t_{2}-r^{2}\right\}}\left(r^{\mu-2}|u|^{2}+r^{\mu+2}|\nabla u|^{2}|\nabla \varphi|^{2}\right) G_{\left(x_{1}, t_{2}\right)} \mathrm{d} x \\
\leq & C r^{\mu}\left(1+r^{2} \rho^{-2}\right) \int_{\mathcal{A} \times\left\{t_{2}-r^{2}\right\}}\left(|\nabla u|^{2}+|u|^{p}\right) G_{\left(x_{1}, t_{2}\right)} \mathrm{d} x \\
& +C \int_{\mathcal{A} \times\left\{t_{2}-r^{2}\right\}} G_{\left(X_{1}, t_{2}\right)} \mathrm{d} x
\end{aligned}
$$

for all $r \leq R_{1}$. Since we can bound

$$
G_{\left(x_{1}, t_{2}\right)} \leq C r^{-n} \mathrm{e}^{-\frac{\rho^{2}}{4 r^{2}}} \text { on } \mathcal{A} \times\left\{t_{2}-r^{2}\right\},
$$

for $0<R \leq \sqrt{t_{2}}$ we have

$$
\begin{aligned}
& \frac{1}{R} \int_{0}^{R}\left|A_{2}^{\varphi}(r)\right| \mathrm{d} r+\int_{0}^{R}\left|A_{0}^{\varphi}(r)\right| r^{-1} \mathrm{~d} r \leq \int_{0}^{R}\left(\left|A_{2}^{\varphi}(r)\right|+\left|A_{0}^{\varphi}(r)\right|\right) r^{-1} \mathrm{~d} r \\
& \quad \leq C \int_{0}^{R} r^{\mu-n-1} \int_{\mathcal{A} \times\left\{t_{2}-r^{2}\right\}}\left(1+\left(\frac{r}{\rho}\right)^{2}\right)\left(|\nabla u|^{2}+|u|^{p}\right) \mathrm{e}^{-\frac{\rho^{2}}{4 r^{2}}} \mathrm{~d} x \mathrm{~d} r+C I,
\end{aligned}
$$

where

$$
I=\int_{0}^{R} \int_{\mathcal{A} \times\left\{t_{2}-r^{2}\right\}} G_{\left(x_{1}, t_{2}\right)} \mathrm{d} x r^{-1} \mathrm{~d} r \leq C \int_{0}^{R}\left(\frac{\rho}{r}\right)^{n} \mathrm{e}^{-\frac{\rho^{2}}{4 r^{2}}} r^{-1} \mathrm{~d} r \leq C\left(\frac{\rho}{R}\right)^{n} \mathrm{e}^{-\frac{\rho^{2}}{4 R^{2}}},
$$

if $R \leq \rho$, and $I \leq C$, else. 
If $R \leq \rho \leq 1$, letting $r_{k}=2^{(1-k) / 2} R, k \in \mathbb{N}$, and substituting $t=t_{2}-r^{2}$, we have

$$
\begin{aligned}
& \int_{0}^{R} r^{\mu-n-1} \int_{\mathcal{A} \times\left\{t_{2}-r^{2}\right\}}\left(1+\left(\frac{r}{\rho}\right)^{2}\right)\left(|\nabla u|^{2}+|u|^{p}\right) \mathrm{e}^{-\frac{\rho^{2}}{4 r^{2}}} \mathrm{~d} x \mathrm{~d} r \\
& \quad \leq 2 \sum_{k=1}^{\infty} \int_{r_{k+1}}^{r_{k}} \int_{\mathcal{A} \times\left\{t_{2}-r^{2}\right\}} r^{\mu-n-2} \mathrm{e}^{-\frac{\rho^{2}}{4 r^{2}}}\left(|\nabla u|^{2}+|u|^{p}\right) \mathrm{d} x r \mathrm{~d} r \\
& \quad \leq C \sum_{k=1}^{\infty} r_{k}^{\mu-n-2} \mathrm{e}^{-\frac{\rho^{2}}{4 r_{k}^{2}}} \int_{\mathcal{A} \times\left[t_{2}-r_{k}^{2}, t_{2}-r_{k+1}^{2}\right]}\left(|\nabla u|^{2}+|u|^{p}\right) \mathrm{d} x \mathrm{~d} t .
\end{aligned}
$$

But by Lemma 6.3 with $t_{1}=t_{2}-r_{k}^{2}, T=t_{2}$, for all $r_{k} \leq 1$, there holds

$$
r_{k}^{\mu-2} \int_{\mathcal{A} \times\left[t_{2}-r_{k}^{2}, t_{2}-r_{k+1}^{2}\right]}\left(|\nabla u|^{2}+|u|^{p}\right) \mathrm{d} x \mathrm{~d} t \leq C .
$$

Hence, if $R \leq \rho \leq 1$, we have

$$
\begin{aligned}
\frac{1}{R} \int_{0}^{R}\left|A_{2}^{\varphi}(r)\right| \mathrm{d} r+\int_{0}^{R}\left|A_{0}^{\varphi}(r)\right| r^{-1} \mathrm{~d} r & \leq C \rho^{-n} \sum_{k=1}^{\infty}\left(\frac{\rho}{r_{k}}\right)^{n} \mathrm{e}^{-\frac{\rho^{2}}{4 r_{k}^{2}}}+C\left(\frac{\rho}{R}\right)^{n} \mathrm{e}^{-\frac{\rho^{2}}{4 R^{2}}} \\
& \leq C\left(1+\rho^{-n}\right)\left(\frac{\rho}{R}\right)^{n} \mathrm{e}^{-\frac{\rho^{2}}{4 R^{2}}} \leq C R^{-n} \mathrm{e}^{-\frac{\rho^{2}}{4 R^{2}}}
\end{aligned}
$$

For the case $R \geq \rho$ we can argue in a similar fashion to bound the remaining integral, also using the fact that, by Lemma 6.3, for any $\rho \leq r_{k} \leq R$ there holds

$$
\int_{\mathcal{A} \times\left[t_{2}-r_{k}^{2}, t_{2}-r_{k+1}^{2}\right]}\left(|\nabla u|^{2}+|u|^{p}\right) \mathrm{d} x \mathrm{~d} t \leq C\left(1+\rho^{2-\mu}+R^{2}\right)
$$

to obtain

$$
\begin{aligned}
& \int_{\rho}^{R} r^{\mu-n-1} \int_{\mathcal{A} \times\left\{t_{2}-r^{2}\right\}}\left(1+\left(\frac{r}{\rho}\right)^{2}\right)\left(|\nabla u|^{2}+|u|^{p}\right) \mathrm{e}^{-\frac{\rho^{2}}{4 r^{2}}} \mathrm{~d} x \mathrm{~d} r \\
& \leq C \rho^{-2} \sum_{k=1}^{k_{0}} r_{k}^{\mu-n} \mathrm{e}^{-\frac{\rho^{2}}{4 r_{k}^{2}}} \int_{\mathcal{A} \times\left[t_{2}-r_{k}^{2}, t_{2}-r_{k+1}^{2}\right]}\left(|\nabla u|^{2}+|u|^{p}\right) \mathrm{d} x \mathrm{~d} t \\
& \leq C\left(1+\rho^{2-\mu}+R^{2}\right) \rho^{-2} \sum_{k=1}^{k_{0}} r_{k}^{\mu-n} \mathrm{e}^{-\frac{\rho^{2}}{4 r_{k}^{2}}} \leq C\left(\rho^{2-\mu}+R^{2}\right) \rho^{\mu-n-2},
\end{aligned}
$$

where $k_{0} \in \mathbb{N}$ is chosen such that $r_{k_{0}+1} \leq \rho \leq r_{k_{0}}$, as claimed. 
Proof of Proposition 6.1. Coupling (6.13) and Lemma 6.4, for any fixed $x_{1} \in \Omega^{\rho}$, any $t_{2} \in$ $\left[T / 2, T\right.$, and any $0<R<R_{1} \leq \sqrt{t_{2}}$, we find

$$
H_{\left(X_{1}, t_{2}\right)}^{\varphi^{\rho}}(R) \leq H_{\left(X_{1}, t_{2}\right)}^{\varphi^{\rho}}\left(R_{1}\right)+C \delta\left(\rho, R_{1}\right)
$$

Passing to the limit $t_{2} \uparrow T$, we conclude the proof of Proposition 6.1.

Proof of Proposition 6.2. Fix any point $x_{0} \in \Omega$ and suppose that $\Omega_{r_{0}}\left(x_{0}\right) \subset \Omega^{\rho}$ and $8 r_{0}^{2} \leq$ $R_{1}^{2} \leq T$. Set $t_{0}=T-r_{0}^{2} \geq 7 T / 8$.

For any $\left.\left(x_{1}, t_{1}\right) \in Q_{r_{0}}\left(x_{0}, t_{0}\right)\right)$ and any $0<R<2 r_{0}<\rho$ we let $t_{2}=t_{1}+R^{2} / 4>t_{0}-r_{0}^{2}=$ $T-2 r_{0}^{2}$. With $\left(x_{1}, t_{2}\right)$ as the center of scaling and observing that $\varphi=\varphi^{\rho}=1$ on $B_{2 r_{0}}\left(x_{1}\right)$, for any $r>0$ with $R / 2<r<R / \sqrt{2}$ we can bound

$$
R^{\mu-n} \int_{\Omega_{r}\left(x_{1}\right) \times\left\{t_{2}-r^{2}\right\}}\left(|\nabla u|^{2}+|u|^{p}\right) \mathrm{d} x \leq C\left(D^{\varphi}(r)+F^{\varphi}(r)\right) .
$$

Integrating in $R / 2<r<R / \sqrt{2}$, substituting $t=t_{2}-r^{2}$, and dividing by $R^{2}$, from (6.12) and Lemma 6.4 we then obtain the bound

$$
\begin{aligned}
R^{\mu-n-2} \int_{Q_{R / 2}\left(x_{1}, t_{2}-R^{2} / 4\right)}\left(|\nabla u|^{2}+|u|^{p}\right) \mathrm{d} x \mathrm{~d} t & \leq \frac{C}{R} \int_{0}^{R / \sqrt{2}}\left(D^{\varphi}(r)+F^{\varphi}(r)\right) \mathrm{d} r \\
& \leq C H_{\left(x_{1}, t_{2}\right)}^{\varphi^{\rho}}(R / \sqrt{2})+C \delta(\rho, R / \sqrt{2}) \\
& \leq C H_{\left(x_{1}, t_{2}\right)}^{\varphi^{\rho}}\left(R_{2}\right)+C \delta\left(\rho, R_{2}\right)
\end{aligned}
$$

for any $R<2 r_{0}<R_{1} \leq \sqrt{T}$, where $0<R_{2}<R_{1}$ is such that $t_{2}-R_{2}^{2}=T-R_{1}^{2}$; in particular,

$$
R_{2}^{2}=R_{1}^{2}-\left(T-t_{2}\right)>R_{1}^{2}-2 r_{0}^{2} \geq 2 r_{0}^{2} \geq R^{2} / 2
$$

Here we also used the bound

$$
\left(\frac{\rho}{R}\right)^{n} \mathrm{e}^{-\frac{\rho^{2}}{2 R^{2}}} \leq C\left(\frac{\rho}{R_{2}}\right)^{n} \mathrm{e}^{-\frac{\rho^{2}}{4 R_{2}^{2}}}
$$

for $0<R / \sqrt{2}<R_{2}$. An estimate similar to (6.7) then gives

$$
R^{\mu-n-2} \int_{Q_{R / 2}\left(x_{1}, t_{1}\right)}\left(|\nabla u|^{2}+|u|^{p}\right) \mathrm{d} x \mathrm{~d} t \leq H_{\left(X_{1}, T\right)}^{\varphi^{\rho}}\left(R_{1}\right)+C \delta\left(\rho, R_{1}\right)
$$


for any $\left.\left(x_{1}, t_{1}\right) \in Q_{r_{0}}\left(x_{0}, t_{0}\right)\right)$ and any $0<R<2 r_{0}$. Thus, we conclude that

$$
\|\nabla u\|_{L^{2, \mu}\left(O_{r_{0}}\left(x_{0}, t_{0}\right)\right)}+\|u\|_{L^{p, \mu}\left(Q_{r_{0}}\left(x_{0}, t_{0}\right)\right)} \leq \sup _{\left|x_{1}-x_{0}\right|<r_{0}} H_{\left(x_{1}, T\right)}^{\varphi^{\rho}}\left(R_{1}\right)+C \delta\left(\rho, R_{1}\right)
$$

analogous to (6.9), as claimed.

\subsection{Characterization of blow-up and tangent cones}

The estimates in the preceding section allow one to characterize blow-up, as follows. First note the following lower bound on the blow-up time of solutions in terms of the $L^{\infty}$-norm of the data.

Proposition 6.5. For given smooth data $u_{0}$ let $u \in C^{\infty}(\Omega \times[0, T[)$ be the unique maximal smooth solution of (1.2), (3.1) as above. Then $T \geq\left\|u_{0}\right\|_{L^{\infty}}^{2-p} /(p-2)$.

Proof. (i) Letting

$$
m(t):=\sup _{\Omega}|u(t)|
$$

by (1.2) we have that $m(t)$ is locally Lipschitz and increases at most at the rate

$$
\mathrm{d} m(t) / \mathrm{d} t \leq m(t)^{p-1}
$$

that is,

$$
m^{2-p}(t) \geq m^{2-p}(0)-t(p-2)
$$

and our claim follows.

As usual, in the definition of the functions $H_{\left(x_{0}, t_{0}\right)}, H_{\left(x_{0}, t_{0}\right)}^{\varphi}$ below we choose the origin so that $x_{0}=0$, and, for given $0<\rho<\rho_{0}$, we let $\varphi=\varphi^{\rho}$.

Proposition 6.6. Let $\Omega$ be convex. For given smooth data $u_{0}$ let $u \in C^{\infty}(\Omega \times[0, T[)$ be the unique maximal smooth solution of (1.2), (3.1).

(i) There is $\varepsilon_{0}>0$ such that, whenever $T<\infty$, for any $0<\rho<\rho_{0}$ we have

$$
\sup _{x_{0} \in \Omega} H_{\left(X_{0}, T\right)}(\sqrt{T}) \geq \liminf _{R \downarrow 0} \sup _{x_{0} \in \Omega} H_{\left(x_{0}, T\right)}^{\varphi}(R) \geq \varepsilon_{0}
$$

(ii) If $T=\infty$, there holds $\|u(t)\|_{H^{1} \cap L^{\infty}(\Omega)} \rightarrow 0$ as $t \rightarrow \infty$. 
Proof. (i) By Proposition 4.1 for any $\delta>0$ there exists $\varepsilon=\varepsilon(\delta)>0$ such that whenever for some $x_{0} \in \Omega$ and $0<R^{2}<t_{0}<T$ there holds $\|u\|_{L^{p, \mu}\left(O_{R}\left(x_{0}, t_{0}\right)\right)}^{p}<\varepsilon$, then we have

$$
\|u\|_{L^{\infty}\left(Q_{R / 2}\left(x_{0}, t_{0}\right)\right)} \leq \delta R^{-2 /(p-2)} .
$$

For a suitable number $\delta>0$, to be determined below, we now fix the associated number $\varepsilon>0$. Suppose that there holds

$$
\limsup \sup _{x_{0} \in \Omega}\|u\|_{L^{p, \mu}\left(O_{r}\left(x_{0}, T-r^{2}\right)\right)}^{p}<\varepsilon
$$

In view of (6.20) then there is $r_{0}>0$ such that, for any $t=T-r^{2}>T-r_{0}^{2}$, we obtain the bound

$$
m(t)=\sup _{\Omega}|u(t)| \leq \delta r^{-2 /(p-2)}
$$

that is, we have

$$
m(t) \leq \delta(T-t)^{-1 /(p-2)} \text { for any } T-r_{0}^{2}<t<T .
$$

But then, for any sufficiently large $t_{0}<T$ from (6.19), after shifting time by $t_{0}$, we obtain

$$
m(t)^{2-p} \geq \delta^{2-p}\left(T-t_{0}\right)-\left(t-t_{0}\right)(p-2) \text { for all } t_{0}<t<T
$$

and the latter is strictly positive at time $t=T$ if $0<\delta<(p-2)^{-1 /(p-2)}$ is fixed sufficiently small, contradicting the maximality of $T$. Thus, we must have

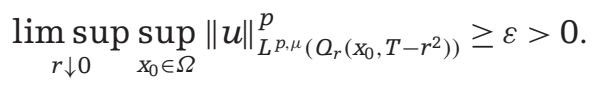

Since $\Omega$ is convex, for any fixed $0<\rho<\rho_{0}$ we have $\Omega^{\rho}=\Omega$. Thus, we may invoke Proposition 6.2 to obtain the bound

$$
0<\varepsilon \leq \lim \sup _{r \downarrow 0} \sup _{x_{0} \in \Omega}\|u\|_{L^{p, \mu}\left(O_{r}\left(x_{0}, T-r^{2}\right)\right)}^{p} \leq C \liminf _{R \downarrow 0} \sup _{x_{0} \in \Omega} H_{\left(x_{0}, T\right)}^{\varphi}(R) .
$$

Moreover, clearly $H_{(x, T)}^{\varphi}(R) \leq H_{(x, T)}(R)$ for any $R>0$ and any $x \in \Omega$. Monotonicity (3.21) then yields the claim. Note that the number $\varepsilon_{0}:=\varepsilon>0$ may be chosen independent of $u$ and $T$. 
(ii) If we assume that $T=\infty$, by (6.6) for any $\varepsilon>0$ we may choose $T_{\varepsilon}>0$ such that, for $t>T_{\varepsilon}$, we have

$$
\sup _{x_{0} \in \Omega} H_{\left(x_{0}, t\right)}(\sqrt{t}) \leq C t^{\frac{\mu-n}{2}}<\varepsilon .
$$

Our claim then follows from (6.9) (with $r_{0}=1$ ) and Proposition 4.1.

In fact, we can characterize the blow-up more precisely.

Lemma 6.7. Let $\Omega$ be convex. Given smooth data $u_{0}$, let $u \in C^{\infty}(\Omega \times[0, T[)$ be the unique maximal smooth solution to (1.2), (3.1). Then there exists a point $x_{0} \in \bar{\Omega}$ such that $H_{\left(X_{0}, T\right)}^{\varphi}(R) \geq \varepsilon_{0} / 2$ for any sufficiently small $\left.R \in\right] 0, \sqrt{T}$, where $\varepsilon_{0}>0$ is as defined in Proposition 6.6.

Proof. By Proposition 6.6, for any fixed $0<\rho<\rho_{0}$ and any sufficiently small $R>0$ there exists $X=x(R) \in \bar{\Omega}$ such that $H_{(x, T)}^{\varphi}(R) \geq \varepsilon_{0}$, where $\varphi=\varphi^{\rho}$. For a suitable sequence $R_{k} \downarrow 0$ then we may assume that $x_{k}=x\left(R_{k}\right) \rightarrow x_{0}$.

We claim that $x_{0}$ has the desired property. Since $\Omega$ is convex and hence $\Omega^{\rho}=\bar{\Omega}$, from Proposition 6.1 for suitably small $R_{1}>0$ we obtain the uniform bound

$$
H_{(X, T)}^{\varphi}(r) \leq H_{(x, T)}^{\varphi}(R)+\varepsilon_{0} / 2
$$

for all $0<r<R<R_{1}$, uniformly for all $x \in \Omega$. Suppose by contradiction that $H_{\left(x_{0}, T\right)}^{\varphi}(R)<$ $\varepsilon_{0} / 2$ for some $0<R<R_{1}$. Fix $k_{0} \in \mathbb{N}$ such that $R_{k}<R$ for $k \geq k_{0}$. Then, for $k \geq k_{0}$ we have

$$
H_{\left(x_{k}, T\right)}^{\varphi}\left(R_{k}\right) \leq H_{\left(x_{k}, T\right)}^{\varphi}(R)+\varepsilon_{0} / 2 \rightarrow H_{\left(X_{0}, T\right)}^{\varphi}(R)+\varepsilon_{0} / 2<\varepsilon_{0} \quad(k \rightarrow \infty),
$$

contradicting our choice of $\left(x_{k}\right)$ for large $k \in \mathbb{N}$.

With the help of Lemma 6.3 we can show the analog of Lemma 6.7 on an arbitrary smooth, bounded domain.

Proposition 6.8. Let $\Omega \subset \mathbb{R}^{n}$ be smooth and bounded. Given smooth data $u_{0}$, let $u \in$ $C^{\infty}(\Omega \times[0, T[)$ be the unique maximal smooth solution to (1.2), (3.1). Then, for any $0<\rho<\rho_{0}$, any $x_{0} \in \Omega^{\rho}$ either

(i) there is $r>0$ such that $u$ extends smoothly to the closure of $Q_{r}\left(x_{0}, T\right)$, or

(ii) for any sufficiently small $R>0$, there holds $H_{\left(x_{0}, T\right)}^{\varphi}(R) \geq \varepsilon_{0}$, where $\varphi=\varphi^{\rho}$ and where $\varepsilon_{0}>0$ is an absolute constant. 
Proof. Fix $0<\rho<\rho_{0}$ and let $x_{0} \in \Omega^{\rho}$. Suppose that, for a sufficiently small number $\varepsilon>0$ to be determined and any sufficiently small $R_{1}>0$, there holds

$$
\sup _{\left|x_{1}-x_{0}\right|<R_{1} ; x_{1} \in \Omega^{\rho}} H_{\left(x_{1}, T\right)}^{\varphi}\left(R_{1}\right) \leq \varepsilon .
$$

By Proposition 6.1, for given $0<\rho<\rho_{0}$ and suitably small $R_{1}>0$ we again have the uniform bound

$$
H_{(X, T)}^{\varphi}(r) \leq H_{(x, T)}^{\varphi}(R)+\varepsilon / 2
$$

for all $0<r<R<R_{1}$, uniformly for all $x \in \Omega^{\rho}$. In particular, in view of our assumption about $x_{0}$ we have

$$
\sup _{\left|x_{1}-x_{0}\right|<R_{1} ; x_{1} \in \Omega^{\rho}} H_{\left(X_{1}, T\right)}^{\varphi}(R)<2 \varepsilon, \quad 0<R<R_{1} .
$$

By Proposition 6.2 then, for any $0<R<R_{1}$, we have

$$
\sup _{\left|x_{1}-X_{0}\right|<R_{1} ; x_{1} \in \Omega^{\rho}}\|u\|_{L^{p, \mu}\left(O_{R / 2}\left(x_{1}, T-R^{2} / 4\right)\right)}^{p}<C \varepsilon .
$$

After scaling for sufficiently small $\varepsilon>0$, Proposition 4.1 implies the bound

$$
(T-t)^{p /(p-2)}\|u(t)\|_{L^{\infty}\left(\Omega_{R_{1} / 2}\left(x_{0}\right)\right)}^{p} \leq C \varepsilon .
$$

We claim that, for sufficiently small $\varepsilon>0$, this bound implies conclusion (i) in the statement of the Lemma. To see this, fix a smooth cutoff function $\eta$ such that $\chi_{B_{R_{1} / 4}\left(x_{0}\right)} \leq$ $\eta \leq \chi_{B_{R_{1} / 2}\left(x_{0}\right)}$. The function $v=v(x, t)=(T-t)^{\alpha} u \eta$ then satisfies the equation

$$
v_{t}-\Delta v=v\left(|u|^{p-2}-\alpha(T-t)^{-1}\right)-f
$$

with

$$
f=2(T-t)^{\alpha} \nabla u \nabla \eta+(T-t)^{\alpha} u \Delta \eta
$$

Multiplying by $2 v$, then we have

$$
\left(\partial_{t}-\Delta\right)|v|^{2}+2|\nabla v|^{2}=2|v|^{2}\left(|u|^{p-2}-\alpha(T-t)^{-1}\right)-2 f v,
$$

where

$$
f v=2(T-t)^{2 \alpha} u \nabla u \eta \nabla \eta+(T-t)^{2 \alpha} u^{2} \eta \Delta \eta
$$


in view of the estimate

$$
|\nabla u| \eta \leq(T-t)^{-\alpha}|\nabla v|+|u||\nabla \eta|
$$

can be bounded

$$
|2 f v(t)| \leq|\nabla v|^{2}+C(T-t)^{2 \alpha} u^{2}\left(\eta \Delta \eta+|\nabla \eta|^{2}\right) .
$$

Thus, we find

$$
\left(\partial_{t}-\Delta\right)|v|^{2}+|\nabla v|^{2}=2|v|^{2}\left(|u|^{p-2}-\alpha(T-t)^{-1}\right)+g
$$

where now

$$
\|g(t)\|_{L^{\infty}\left(\Omega_{R_{1} / 2}\left(x_{0}\right)\right)} \leq C(T-t)^{2 \alpha}\|u(t)\|_{L^{\infty}\left(\Omega_{R_{1} / 2}\left(x_{0}\right)\right)}^{2} \leq C(T-t)^{2 \alpha-\frac{2}{p-2}} .
$$

For sufficiently large $0 \leq \alpha<1 /(p-2)$ then we see that $\|g(t)\|_{L^{\infty}\left(\Omega_{\left.R_{1} / 2\left(x_{0}\right)\right)}\right.} \in L^{1}$ ([t $t_{0}, T[)$ for some $t_{0}<T$, while (6.24) for sufficiently small $\varepsilon>0$ implies that $|u|^{p-2} \leq \alpha(T-t)^{-1}$ on $\Omega_{R_{1} / 2}\left(x_{0}\right) \times\left[t_{0}, T\left[\right.\right.$. The maximum principle then gives a uniform bound for $v$ on $\left[t_{0}, T[\right.$; hence

$$
(T-t)^{\alpha p}\|u(t)\|_{L^{\infty}\left(\Omega_{R_{1} / 4}\left(x_{0}\right)\right)}^{p} \leq C \quad \text { for } t_{0}<t<T .
$$

By iteration, in finitely many steps we can achieve the bound

$$
(T-t)^{1 / 2}\|u(t)\|_{L^{\infty}\left(\Omega_{R_{1} / 8}\left(x_{0}\right)\right)}^{p} \leq C \quad \text { for } t_{1}<t<T .
$$

Repeating the above argument with $\alpha=0$, we see that $u \in L^{\infty}\left(Q_{R_{1} / 9}\left(x_{0}, T\right)\right)$. By parabolic regularity then $u$ smoothly extends to the closure of $Q_{R_{1} / 10}\left(x_{0}, T\right)$ ).

Negating (6.22), for any sufficiently small $R>0$ we can find $x=x(R) \in \Omega^{\rho}$ such that $\left|x-x_{0}\right|<R$ and $H_{(x, T)}^{\varphi}(R) \geq \varepsilon$. We may then proceed as in the proof of Lemma 6.7. For a suitable sequence $R_{k} \downarrow 0$ we let $x_{k}=x\left(R_{k}\right) \rightarrow x_{0}$. It is now easy to show that $x_{0}$ has the desired property. Indeed, suppose by contradiction that $H_{\left(X_{0}, T\right)}^{\varphi}(R)<\varepsilon / 2=$ : $\varepsilon_{0}$ for some $0<R<R_{1}$. Arguing as above, we may assume that $R_{1}>0$ is chosen such that (6.23) holds. Fix $k_{0} \in \mathbb{N}$ such that $R_{k}<R$ for $k \geq k_{0}$. Then, for $k \geq k_{0}$ by (6.23) we have

$$
H_{\left(x_{k}, T\right)}^{\varphi}\left(R_{k}\right) \leq H_{\left(x_{k}, T\right)}^{\varphi}(R)+\varepsilon / 2 \rightarrow H_{\left(X_{0}, T\right)}^{\varphi}(R)+\varepsilon / 2<\varepsilon \quad(k \rightarrow \infty),
$$

contradicting our choice of $\left(x_{k}\right)$ for large $k \in \mathbb{N}$.

Theorem 6.9. Let $\Omega \subset \mathbb{R}^{n}$ be smooth and bounded. For given smooth data $u_{0}$ let $u \in$ $C^{\infty}\left(\Omega \times\left[0, T[)\right.\right.$ be the unique maximal smooth solution to (1.2), (3.1). Let $\left(x_{0}, T\right)$ be a first 
blow-up point in the sense of Lemma 6.8(ii), where $x_{0} \in \Omega$. (If $\Omega$ is convex, we also allow $x_{0} \in \partial \Omega$.) Shift coordinates so that $\left(x_{0}, T\right)=(0,0)$. Then the following holds:

(i) There exists a sequence $R_{k} \rightarrow 0$ and a self-similar "ancient" weak solution $\bar{u}$ for all $R>0$ on $\mathbb{R}^{n} \times$ ] $-\infty, 0\left[\right.$ (or on the half-space $\left.\mathbb{R}_{+}^{n} \times\right]-\infty, 0[$ ) which is smooth away from a set $\operatorname{sing}(\bar{u})$ of locally finite $(n+2-\mu)$-dimensional Hausdorff measure such that $u_{k}=u_{R_{k}} \rightarrow \bar{u}$ as $k \rightarrow \infty$, smoothly locally away from $\operatorname{sing}(\bar{u})$ and from $t=0$. (Recall that $\bar{u}$ is self-similar if $\bar{u} \equiv \bar{u}_{R}$ for all $R>0$, where, for any $R>0$, we let $u_{R}(x, t)=R^{2 /(p-2)} u\left(R x, R^{2} t\right)$.)

If $\left(x_{0}, T\right)=(0,0)$ is of Type $I$ in the sense that there exist constants $C, r_{0}>0$ such that

$$
\limsup _{t \uparrow 0}\left(|t|^{1 /(p-2)} \sup _{x \in \Omega_{r_{0}}(0)}|u(x, t)|\right) \leq C,
$$

then $\bar{u} \neq 0, \bar{u}$ is smooth, and $\left(u_{k}\right)$ converges smoothly to $\bar{u}$, locally away from $t=0$.

(ii) If $\left(x_{0}, T\right)=(0,0)$ is not of Type $I$ in addition to the above, there exist sequences $R_{k} \rightarrow 0, t_{k} \uparrow 0, \Omega \ni x_{k} \rightarrow 0$, and an "eternal" solution $\bar{u} \neq 0$ of 1.2 on $\mathbb{R}^{n} \times \mathbb{R}$ (or possibly on the half-space $\mathbb{R}_{+}^{n} \times \mathbb{R}$ if $\Omega$ is convex and $x_{0} \in \partial \Omega$ ) with

$$
\|\bar{u}\|_{L^{\infty}}=|\bar{u}(0,0)|=1
$$

and such that

$$
u_{k}(x, t)=R_{k}^{\frac{2}{p-2}} u\left(x_{k}+R_{k} x, t_{k}+R_{k}^{2} t\right) \rightarrow \bar{u}
$$

smoothly locally as $k \rightarrow \infty$.

Proof. Let $x_{0} \in \Omega$ with $H_{\left(x_{0}, T\right)}^{\varphi}(R) \geq \varepsilon>0$ for any sufficiently small $R>0$ and some uniform number $\varepsilon>0$. Shift $\left(x_{0}, T\right)=(0,0)$. Since $\Omega$ is open, there exists $0<\rho<\rho_{0}$ such that $x_{0} \in \Omega^{\rho}$. (Recall that, for any convex domain, we have $\Omega^{\rho}=\bar{\Omega}$ for any $0<\rho<\rho_{0}$.)

(i) If $\left(x_{0}, T\right)=(0,0)$ satisfies $(6.25)$, we may assume that $\rho<r_{0} / 4$. Moreover, by assumption (6.25) there is $\tau>0$ such that, for any $R>0$, the rescaled solution $u_{R}$ is uniformly locally bounded on the domain $\left.Q_{R}:=\Omega_{r_{0} / R}(0) \times\right]-\tau / R^{2}$, O[ with

$$
\sup _{-\tau / R^{2}<t<0,|x| \leq r_{0} / R}|t|^{\frac{1}{p-2}}\left|u_{R}(x, t)\right|<\infty
$$


and $u_{R}$ solves (1.2) on $Q_{R}$. By parabolic regularity then, after scaling, for any sufficiently small $R>0$ we also have the bound

$$
\sup _{-\frac{\tau}{2 R^{2}}<t<0,|x| \leq r_{0}}|t|^{\frac{p}{p-2}}\left|\nabla u_{R}(X, t)\right|^{2} \leq C .
$$

Therefore, a subsequence $u_{k}=u_{R_{k}}$ converges smoothly locally on $\left.\mathbb{R}^{n} \times\right]-\infty$, $0\left[\right.$ to a $C^{1}$ solution $\bar{u}$ of (1.2) on $\left.\mathbb{R}^{n} \times\right]-\infty$, 0[. (Similarly, if $\Omega$ is convex and $x_{0} \in \partial \Omega$, a subsequence $u_{k}=u_{R_{k}}$ converges smoothly locally on a half-space $\left.\mathbb{R}_{+}^{n} \times\right]-\infty, 0[$.)

Moreover, from (3.18), (3.19), Lemma 6.4, and (6.12), upon integrating in $R$ and substituting $t=-R^{2}$, for any $R_{0}>0$ with $\varphi=\varphi^{\rho}$ we obtain

$$
\begin{aligned}
H^{\varphi}\left(R_{0}\right) & \geq H^{\varphi}\left(R_{0}\right)-\liminf _{R \rightarrow 0} H^{\varphi}(R)=\int_{0}^{R_{0}} \frac{\mathrm{d}}{\mathrm{d} R} H^{\varphi}(R) \mathrm{d} R \\
& \geq \int_{0}^{R_{0}} \frac{R^{\mu-4}}{2 p} \int_{\Omega \times\left\{-R^{2}\right\}}\left|X \cdot \nabla u+2 t u_{t}+a u\right|^{2} \varphi^{2} G \mathrm{~d} x R \mathrm{~d} R+I_{0}^{\varphi} \\
& =\frac{1}{4 p} \int_{-R_{0}^{2}}^{0} \int_{\Omega}|t|^{a-1}\left|X \cdot \nabla u+2 t u_{t}+a u\right|^{2} \varphi^{2} G \mathrm{~d} x \mathrm{~d} t+I_{0}^{\varphi},
\end{aligned}
$$

where $a=\frac{2}{p-2}$ and where in view of Lemma 6.4 we have

$$
I_{0}^{\varphi}=\frac{2}{p} \int_{0}^{R_{0}} R^{-1} A_{0}^{\varphi}(R) \mathrm{d} R \leq C \delta\left(\rho, R_{0}\right) \rightarrow 0 \quad\left(R_{0} \downarrow 0\right) .
$$

Therefore, we find

$$
\begin{aligned}
& \int_{-\infty}^{0} \int_{\mathbb{R}^{n}}|t|^{a-1}\left|X \cdot \nabla \bar{u}+2 t \bar{u}_{t}+a \bar{u}\right|^{2} G \mathrm{~d} x \mathrm{~d} t \\
& \quad=\lim _{L \rightarrow \infty} \int_{-L^{2}}^{-1 / L^{2}} \int_{B_{L}(0)}|t|^{a-1}\left|X \cdot \nabla \bar{u}+2 t \bar{u}_{t}+a \bar{u}\right|^{2} G \mathrm{~d} x \mathrm{~d} t \\
& \quad \leq \lim _{L \rightarrow \infty} \limsup _{R \rightarrow 0} \int_{-L^{2}}^{-1 / L^{2}} \int_{B_{L}(0)}|t|^{a-1}\left|X \cdot \nabla u_{R}+2 t u_{R, t}+a u_{R}\right|^{2} G \mathrm{~d} x \mathrm{~d} t \\
& \quad=\lim _{L \rightarrow \infty} \limsup _{R \rightarrow 0} \int_{-L^{2} R^{2}}^{(R / L)^{2}} \int_{B_{L R}(0)}|t|^{a-1}\left|X \cdot \nabla u+2 t u_{t}+a u\right|^{2} \varphi^{2} G \mathrm{~d} x \mathrm{~d} t=0,
\end{aligned}
$$

and we conclude that $\frac{\mathrm{d} \bar{u}_{R}}{\mathrm{~d} R} \equiv 0$, so that $\bar{u}=\bar{u}_{R}$ for any $R>0$. Similarly, from (6.26), (6.27) it follows that

$$
0<\varepsilon \leq \liminf _{R \downarrow 0} H_{u}^{\varphi}(R) \leq H_{u}^{\varphi}\left(R_{k}\right)=H_{u_{R_{k}}}^{\varphi_{R_{k}}}(1) \rightarrow H_{\bar{u}}(1) \quad \text { as } k \rightarrow \infty ;
$$

hence $\bar{u} \neq 0$, as claimed. 
If $\left(x_{0}, T\right)=(0,0)$ is not of Type I, we can still show existence and partial regularity of $\bar{u}$, as follows. For any compact $Q \subset \mathbb{R}^{n} \times$ ] $-\infty$, 0 [ we can find finitely many parabolic cylinders $P_{r_{i}}\left(y_{i}, s_{i}\right)$ such that $Q \subset \cup_{i} P_{r_{i}}\left(y_{i}, s_{i}\right)$ and $s_{i}+r_{i}^{2}=0$. For any fixed $R_{1}>0$ and sufficiently small $R>0$ with $2 \max _{i} R r_{i}<R_{1}$, by Proposition 6.2 we have

$$
\begin{aligned}
\left\|\nabla u_{R}\right\|_{L^{2, \mu}\left(P_{r_{i}}\left(y_{i}, s_{i}\right)\right)}^{2}+\left\|u_{R}\right\|_{L^{p, \mu}\left(P_{r_{i}}\left(Y_{i}, S_{i}\right)\right)}^{p}= & \|\nabla u\|_{L^{2, \mu}\left(P_{r_{i} R}\left(R Y_{i}, R^{2} s_{i}\right)\right)}^{2} \\
& +\|u\|_{L^{p, \mu}\left(P_{r_{i} R}\left(R_{Y_{i}}, R^{2} s_{i}\right)\right)}^{p} \leq C\left(R_{1}\right)<\infty .
\end{aligned}
$$

Hence we obtain that

$$
\left\|\nabla u_{R}\right\|_{L^{2}(Q)}+\left\|u_{R}\right\|_{L^{p}(Q)} \leq C(Q)<\infty
$$

uniformly for sufficiently small $R>0$, and we may assume that, for a sequence $R_{k} \rightarrow 0$, the rescaled functions $u_{k}=u_{R_{k}} \rightarrow \bar{u}$ locally weakly in $L^{p}$ on $\left.\mathbb{R}^{n} \times\right]-\infty, 0[$ as $k \rightarrow \infty$ with $\nabla u_{k} \rightarrow \nabla \bar{u}$ locally weakly in $L^{2}$, where $\bar{u}$ solves (1.2).

For a suitable number $\varepsilon>0$ to be determined and any given compact set $Q \subset$ $\left.\mathbb{R}^{n} \times\right]-\infty, 0[$ define

$$
S=\left\{z=(y, s) \in Q ; \liminf _{r \downarrow 0} \liminf _{k \rightarrow \infty} r^{\mu-n-2} \int_{S_{r}(z)}\left(\left|\nabla u_{k}\right|^{2}+\left|u_{k}\right|^{p}\right) \mathrm{d} z^{\prime}>\varepsilon\right\},
$$

where for any $z_{0}=\left(x_{0}, t_{0}\right)$ in our new coordinates and any $r>0$ now

$$
S_{r}\left(z_{0}\right)=\left\{z=(x, t) ;\left|x-x_{0}\right|<r,\left|t-t_{0}\right|<r^{2}\right\}
$$

is a centered parabolic cylinder.

Let $r_{Q}>0$ such that $26 r_{Q}^{2}+t<0$ for all $z=(x, t) \in Q$. For any given $0<r_{0}<r_{Q}$ and each $z_{0}=\left(x_{0}, t_{0}\right) \in S$ there is $0<r=r\left(z_{0}\right)<r_{0}$ such that

$$
r^{\mu-n-2} \int_{S_{r}\left(z_{0}\right)}\left(\left|\nabla u_{k}\right|^{2}+\left|u_{k}\right|^{p}\right) \mathrm{d} z \geq \varepsilon
$$

for all sufficiently large $k \in \mathbb{N}$.

The family $\left(S_{r(z)}(z)\right)_{z \in S}$ covers $S$. By Vitali's covering lemma (see Caffarelli et al. [4, Lemma 6.1, p. 806], for a parabolic version) there exists a collection $z_{i}=\left(y_{i}, s_{i}\right) \in S, i \in \mathbb{N}$, such that the cylinders $S_{i}=S_{r_{i}}\left(z_{i}\right)$ are disjoint while $S \subset \cup_{i \in \mathbb{N}} S_{i}^{*}$, where $r_{i}=r\left(z_{i}\right)$ and where 
$S_{i}^{*}=S_{5 r_{i}}\left(z_{i}\right), i \in \mathbb{N}$. Since $z_{i} \in S \subset Q$, we have $S_{i}^{*} \subset U_{5 r_{Q}}(Q):=\cup_{z \in Q} S_{5 r_{Q}}(z)$, and by assumption $r_{Q}>0$ is so small that the closure $Q_{0}$ of $U_{5 r_{Q}}(Q)$ is contained in $\left.\mathbb{R}^{n} \times\right]-\infty, 0[$. Hence we can estimate

$$
\begin{aligned}
\varepsilon \sum_{i \in \mathbb{N}} r_{i}^{n+2-\mu} & \leq \int_{\bigcup_{i \in \mathbb{N}} S_{i}}\left(\left|\nabla u_{k}\right|^{2}+\left|u_{k}\right|^{p}\right) \mathrm{d} z \leq \int_{\bigcup_{i \in \mathbb{N}} S_{i}^{*}}\left(\left|\nabla u_{k}\right|^{2}+\left|u_{k}\right|^{p}\right) \mathrm{d} z \\
& \leq \int_{Q_{0}}\left(\left|\nabla u_{k}\right|^{2}+\left|u_{k}\right|^{p}\right) \mathrm{d} z \leq C<\infty
\end{aligned}
$$

with a uniform constant independent of $r_{0}$. Hence $\mathcal{H}^{n+2-\mu}(S) \leq C$. In particular, we note that the $(n+1)$-dimensional measure of the cover $\cup_{i \in \mathbb{N}} S_{i}^{*}$ of $S$ converges to 0 as $r_{0} \rightarrow 0$. Thus, in fact, $\mathcal{H}^{n+2-\mu}(S)=0$.

To complete the proof in case (i) observe that, for any $z_{0}=\left(x_{0}, t_{0}\right) \in Q \backslash S$ and any $0<r_{0}<\rho_{0}$, with a number $L \in \mathbb{N}$ to be determined below by Proposition 6.1, (6.3), Fubini's theorem, and the definition of $S$, there exists $0<r<r_{0} / 3$ such that, with $\varphi=\varphi^{r}$ for a sequence $k \rightarrow \infty$, there holds

$$
\begin{aligned}
& \sup _{\left|x_{1}-x_{0}\right|<r} H_{u_{k},\left(x_{1}, t_{0}+2 r^{2} / L^{2}\right)}^{\varphi}(2 r / L) \\
& \leq C \inf _{r / L<s<2 r / L} s^{\mu-n} \int_{B_{3 r}\left(x_{0}\right) \times\left\{t_{0}-2 s^{2}\right\}}\left(\left|\nabla u_{k}\right|^{2}+\left|u_{k}\right|^{p}\right) \mathrm{d} x+C \delta(r, 2 r / L) \\
& \leq C L^{n+2-\mu} r^{\mu-n-2} \int_{S_{3 r}\left(z_{0}\right)}\left(\left|\nabla u_{k}\right|^{2}+\left|u_{k}\right|^{p}\right) \mathrm{d} z+C \delta(\rho, 2 r / L) \\
& \leq C L^{n+2-\mu} \varepsilon+C \delta(r, 2 r / L) .
\end{aligned}
$$

Setting $R_{1}=2 r / L=2 r_{0}, T=t_{0}+2 r^{2} / L^{2}$ in Proposition 6.2, upon fixing $L \in \mathbb{N}$ sufficiently large to guarantee that $\delta(r, 2 r / L) \leq L^{n+2-\mu} \varepsilon$, we find $\left\|u_{k}\right\|_{L^{p, \mu}\left(P_{r_{0}}\left(z_{0}\right)\right)} \leq C L^{n+2-\mu} \varepsilon$. Choosing $\varepsilon>0$ sufficiently small, from Proposition 4.1 we then deduce a uniform bound for $\left(u_{k}\right)$ in a neighborhood of $z_{0}$.

Exhausting the domain $\mathbb{R}^{n} \times$ ] $-\infty$, 0[ by compact regions $Q=Q_{k}$ as above and passing to a suitable diagonal subsequence $\left(u_{k}\right)$, we obtain the claim.

ii) If (after shifting time by $T)\left(x_{0}, T\right)=(0,0)$ is not of Type $\mathrm{I}$, for all $r>0$ we have

$$
\limsup _{t \uparrow 0}|t|^{\frac{1}{p-2}} \sup _{x \in \Omega_{r}(0)}|u(x, t)|=\infty
$$


Following Hamilton [16], for $k \in \mathbb{N}$ and any $r>0$ we let $\left(x_{k}, t_{k}\right) \in \Omega_{r} \times\left[-T,-\frac{1}{k}\right]$ such that

$$
\begin{aligned}
M_{k} & :=\sup _{(x, t) \in \Omega_{r} \times\left[-T,-\frac{1}{k}\right]}|u(x, t)|\left(-\frac{1}{k}-t\right)^{\frac{1}{p-2}}(r-|x|)^{\frac{2}{p-2}} \\
& =\left|u\left(x_{k}, t_{k}\right)\right|\left(-\frac{1}{k}-t_{k}\right)^{\frac{1}{p-2}}\left(r-\left|x_{k}\right|\right)^{\frac{2}{p-2}}
\end{aligned}
$$

where $\Omega_{r}=\Omega_{r}(0)$ for brevity.

Since for any $(x, t)$ we have

$$
\lim _{k \rightarrow \infty}|u(x, t)|\left(-\frac{1}{k}-t\right)^{\frac{1}{p-2}}=|u(x, t)||t|^{\frac{1}{p-2}}
$$

from our assumption we deduce that $M_{k} \rightarrow \infty$ and $t_{k} \uparrow 0$ as $k \rightarrow \infty$.

Set $R_{k}:=\left|u\left(x_{k}, t_{k}\right)\right|^{\frac{2-p}{2}}>0$ and scale

$$
u_{k}(x, t)=R_{k}^{\frac{2}{p-2}} u\left(x_{k}+R_{k} X, t_{k}+R_{k}^{2} t\right)=\frac{u\left(x_{k}+R_{k} X, t_{k}+R_{k}^{2} t\right)}{\left|u\left(x_{k}, t_{k}\right)\right|}
$$

Note that as $k \rightarrow \infty$, we have

$$
R_{k}^{-2}\left(-\frac{1}{k}-t_{k}\right)\left(r-\left|x_{k}\right|\right)^{2}=\left(\left|u\left(x_{k}, t_{k}\right)\right|\left(-\frac{1}{k}-t_{k}\right)^{\frac{1}{p-2}}\left(r-\left|x_{k}\right|\right)^{\frac{2}{p-2}}\right)^{p-2} \rightarrow \infty
$$

and hence

$$
R_{k}^{-2}\left(-\frac{1}{k}-t_{k}\right), \quad R_{k}^{-1}\left(r-\left|x_{k}\right|\right) \rightarrow \infty
$$

For $(x, t) \in \mathbb{R}^{n} \times \mathbb{R}$ and any sufficiently large $k \in \mathbb{N}$ so that $R_{k}^{-1}\left(r-\left|x_{k}\right|\right) \geq|x|$ and $t \in\left[-R_{k}^{-2}\left(T+t_{k}\right), R_{k}^{-2}\left(-\frac{1}{k}-t_{k}\right)\right]$ in view of (6.28) as $k \rightarrow \infty$, we then find

$$
\begin{aligned}
\left|u_{k}(x, t)\right| & =\frac{\left|u\left(x_{k}+R_{k} x, t_{k}+R_{k}^{2} t\right)\right|}{\left|u\left(x_{k}, t_{k}\right)\right|} \\
& \leq\left(\frac{-\frac{1}{k}-t_{k}}{-\frac{1}{k}-t_{k}-R_{k}^{2} t}\right)^{\frac{1}{p-2}}\left(\frac{r-\left|x_{k}\right|}{r-\left|x_{k}+R_{k} x\right|}\right)^{\frac{2}{p-2}} \\
& \leq\left(\frac{R_{k}^{-2}\left(-\frac{1}{k}-t_{k}\right)}{R_{k}^{-2}\left(-\frac{1}{k}-t_{k}\right)-t}\right)^{\frac{1}{p-2}}\left(\frac{R_{k}^{-1}\left(r-\left|x_{k}\right|\right)}{R_{k}^{-1}\left(r-\left|x_{k}\right|\right)-|x|}\right)^{\frac{2}{p-2}} \rightarrow 1
\end{aligned}
$$

uniformly on compact subsets of $\mathbb{R}^{n} \times \mathbb{R}$. 
For sequences $r_{m} \downarrow 0, L_{m} \rightarrow \infty$ by the argument above we can then find a sequence of points $\left(x_{m}, t_{m}\right) \in \Omega_{r_{m}} \times\left[-\frac{1}{m}, 0\right)$ and radii $0<R_{m}<L_{m}^{-1} \rightarrow 0$ such that the rescaled solutions

$$
u_{m}(x, t)=R_{m}^{\frac{2}{p-2}} u\left(x_{m}+R_{m} x, t_{m}+R_{m}^{2} t\right)=\frac{u\left(x_{m}+R_{m} x, t_{m}+R_{m}^{2} t\right)}{\left|u\left(x_{m}, t_{m}\right)\right|}
$$

solve (1.2) on $B_{L_{m}}(0) \times\left[-L_{m}, L_{m}\right]$ with $\left|u_{m}(0,0)\right|=1$ and such that

$$
\left\|u_{m}\right\|_{L^{\infty}\left(B_{L_{m}}(0) \times\left[-L_{m}, L_{m}\right]\right)} \leq 1+\frac{1}{m} .
$$

Hence, $\left(u_{m}\right)$ converges smoothly locally to an "eternal" solution $\bar{u}$ of (1.2) on $\mathbb{R}^{n} \times \mathbb{R}$ as $k \rightarrow \infty$, which satisfies

$$
\|\bar{u}\|_{L^{\infty}}=1=|\bar{u}(0,0)|
$$

Remark 6.10. In certain situations the possible blow-up profiles $\bar{u}$ arising in Theorem 6.9 have been classified completely. Giga and Kohn [15] showed that a Type I blow-up, as defined in Theorem 6.9, in the subcritical and critical cases $p \leq 2^{*}$ always is associated with a spatially constant profile. Moreover, Merle and Zaag [23, 24] established that in the subcritical case non-negative solutions $u$ of (1.2) on a convex domain can only have blow-up of Type I and that ancient solutions $u$ on $\left.\mathbb{R}^{n} \times\right]-\infty, T$ ] necessarily are of ode-type (spatially constant). For $p>2^{*}$, however, examples of self-similar Type I blow-up with spatially nonconstant profile were obtained by Troy [36], and Herrero and Velazquez [17] demonstrated that, for sufficiently large $n$ and $p$, also a Type II blow-up may occur. Matano and Merle [20-22] and Mizoguchi [25] characterize the different possible blow-up regimes in the radially symmetric case. (See [22] for a more exhaustive list of references.)

If we assume that $\bar{u}=\bar{u}(x)>0$ is a time-independent, self-similar solution of (1.1) on $\mathbb{R}^{n}$, that is, satisfying $\bar{u} \equiv \bar{u}_{R}$ for all $R>0$, and if we suppose that in case $n>3$ we have $p<2 \frac{n-1}{n-3}$, the critical Sobolev exponent in $n-1$ dimensions, a result of Gidas and Spruck [14], implies that $\bar{u}(x)=C_{*}|x|^{-a}$, where $C_{*}=(a(n-2-a))^{\frac{1}{p-2}}$; see [38] or [32] for details. (Any exponent $p>2 *$ is admissible when $n=3$.)

\subsection{Well-posedness in the Morrey space}

The estimates in the preceding section allow one to considerably relax the regularity assumptions on the data. For instance, Proposition 6.5 shows that the Cauchy problem (1.2), (3.1) is locally well-posed for initial data $u_{0} \in L^{\infty}(\Omega)$. 
Moreover, if the domain is convex, and if $u_{0} \in H^{1} \cap L^{p, \mu}(\Omega)$ with $\nabla u_{0} \in L^{2, \mu}(\Omega)$ for sufficiently small $\varepsilon>0$ satisfies the condition

$$
\sup _{x_{0} \in R^{n}, 0<r<r_{0}} r^{\lambda-n} \int_{\Omega_{r}\left(x_{0}\right) \cap \Omega}\left(\left|\nabla u_{0}\right|^{2}+\left|u_{0}\right|^{p}\right) d x<\varepsilon
$$

for some number $r_{0}=r_{0}(\varepsilon)>0$, then (6.9) and Proposition 4.1 give the a priori $L^{\infty}$-bound

$$
\left\|u_{k}(t)\right\|_{L^{\infty}(\Omega)} \leq C t^{-\frac{2}{p-2}}
$$

on a uniform time interval $0<t<T$ for the solutions $u_{k}$ of (1.2) for suitable smooth data $u_{0 k} \rightarrow u_{0}$ in $H^{1} \cap L^{p}(\Omega)(k \rightarrow \infty)$ satisfying the bound (6.29). Thus, we can extend our results to data $u_{0}$ in this class.

The latter reasoning fails, however, when the domain is nonconvex, and it is not clear if the Cauchy problem (1.2), (3.1) is locally well-posed on an arbitrary smooth domain for small data in this class. More generally, we may ask if the Cauchy problem is locally well-posed even for arbitrarily large initial data $u_{0} \in H^{1} \cap L^{p, \mu}(\Omega)$ with $\nabla u_{0} \in$ $L^{2, \mu}(\Omega)$. See [3] for a related study.

Concerning the time-independent problem, it seems an interesting question if any (not necessarily stationary) weak solution $u \in H^{1} \cap L^{p, \mu}(\Omega)$ of (1.1) with $\nabla u \in L^{2, \mu}(\Omega)$ is partially regular in the sense of Pacard. Likewise, we may ask if Pacard's partial regularity result [28, Proposition 1] and our improvement of this result in Proposition 5.1 extend up to the boundary of the domain when the domain is nonconvex.

\section{Acknowledgement}

We thank Frank Merle for bringing to our attention some further references relevant for our topic.

\section{Funding}

S.B. gratefully acknowledges the support of the Forschungsinstitut für Mathematik (FIM), ETH Zurich and of the Swiss National Science Foundation under grant no. 200020-125127. M.S. would like to thank the Courant Institute of NYU, Stanford University, and the Hausdorff Center of Bonn University for hospitality and support.

\section{References}

[1] Adams, D. R. "A note on Riesz potentials." Duke Mathematical Journal 42, no. 4 (1975): 765-78. 
[2] Brezis, H. Elliptic Equations with Limiting Sobolev Exponents: The Impact of Topology. Frontiers of the Mathematical Sciences: 1985. New York, 1985; Communications on Pure and Applied Mathematics 39 (1986): S17-39.

[3] Brezis, H. and T. Cazenave. "A nonlinear heat equation with singular initial data." Journal d'Analyse Mathématique 68 (1996): 277-304.

[4] Caffarelli, L., R. Kohn, and L. Nirenberg. "Partial regularity of suitable weak solutions of the Navier-Stokes equations." Communications on Pure and Applied Mathematics 35, no. 6 (1982): 771-831.

[5] Chou, K.-S., S.-Z. Du, and G.-F. Zheng. "On partial regularity of the borderline solution of semilinear parabolic problems." Calculus of Variations and Partial Differential Equations 30, no. 2 (2007): 251-75.

[6] Dancer, E. N. and J. Wei. "Sign-changing solutions for supercritical elliptic problems in domains with small holes." Manuscripta Mathematica 123, no. 4 (2007): 493-511.

[7] del Pino, M., P. Felmer, and M. Musso. "Two-bubble solutions in the super-critical BahriCoron's problem." Calculus of Variations and Partial Differential Equations 16, no. 2 (2003): $113-45$.

[8] del Pino, M. and J. Wei. "Problèmes elliptiques supercritiques dans des domaines avec de petits trous." (English, French summary) [Supercritical elliptic problems in domains with small holes]. Annales de l'Institut Henri Poincaré. Analyse Non Linéaire 24, no. 4 (2007): 507-20.

[9] Evans, L. C. "Partial regularity for stationary harmonic maps into spheres." Archive for Rational Mechanics and Analysis 116, no. 2 (1991): 101-13.

[10] Friedman, A. and B. McLeod. "Blow-up of positive solutions of semilinear heat equations." Indiana University Mathematics Journal 34, no. 2 (1985): 425-47.

[11] Fujita, H. "On the blowing up of solutions of the Cauchy Problem for $u_{t}=\Delta u+u^{1+\alpha}$." J. Fac. Sci. Univ. Tokyo Sect. I 13 (1996): 109-24.

[12] Gazzola, F., H.-C. Grunau, G. Sweers. "Polyharmonic Boundary Value Problems." Positivity Preserving and Nonlinear Higher Order Elliptic Equations in Bounded Domains. Lecture Notes in Mathematics, 1991. Berlin: Springer, 2010.

[13] Ge, Y., R. Jing, and F. Pacard. "Bubble towers for supercritical semilinear elliptic equations." Journal of Functional Analysis 221, no. 2 (2005): 251-302.

[14] Gidas, B. and J. Spruck. "Global and local behavior of positive solutions of nonlinear elliptic equations." Communications on Pure and Applied Mathematics 34, no. 4 (1981): 525-98.

[15] Giga, Y. and R. V. Kohn. "Asymptotically self-similar blow-up of semilinear heat equations." Communications on Pure and Applied Mathematics 38, no. 3 (1985): 297-319.

[16] Hamilton, R. S. "Three-manifolds with positive Ricci curvature." Journal of Differential Geometry 17, no. 2 (1982): 255-306.

[17] Herrero, M. A. and J. J. L. Velázquez. “Explosion de solutions d'équations paraboliques semilinéaires supercritiques." [Blowup of solutions of supercritical semilinear parabolic equations] Comptes Rendus de l'Académie des Sciences Series I-Mathematics 319, no. 2 (1994): 141-5. 
[18] Huisken, G. "Asymptotic behavior for singularities of the mean curvature flow." Journal of Differential Geometry 31, no. 1 (1990): 285-99.

[19] Kaplan, S. "On the growth of solutions of quasi-linear parabolic equations." Communications on Pure and Applied Mathematics 16 (1963): 305-30.

[20] Matano, H. and F. Merle. “On nonexistence of type II blowup for a supercritical nonlinear heat equation." Communications on Pure and Applied Mathematics 57, no. 11 (2004): 1494-541.

[21] Matano, H. and F. Merle. "Classification of type I and type II behaviors for a supercritical nonlinear heat equation." Journal of Functional Analysis 256, no. 4 (2009): 992-1064.

[22] Matano, H. and F. Merle. "Threshold and generic type I behaviors for a supercritical nonlinear heat equation." Journal of Functional Analysis 261, no. 3 (2011): 716-48.

[23] Merle, F. and H. Zaag. "Optimal estimates for blowup rate and behavior for nonlinear heat equations." Communications on Pure and Applied Mathematics 51, no. 2 (1998): 139-96.

[24] Merle, F. and H. Zaag. "A Liouville theorem for vector-valued nonlinear heat equations and applications." Mathematische Annalen 316, no. 1 (2000): 103-37.

[25] Mizoguchi, N. "Rate of type II blowup for a semilinear heat equation." Mathematische Annalen 339, no. 4 (2007): 839-77.

[26] Molle, R. and D. Passaseo. "Positive solutions of slightly supercritical elliptic equations in symmetric domains." Annales de l'Institut Henri Poincaré. Analyse Non Linéaire 21, no. 5 (2004): 639-56.

[27] Ni, W.-M., P. E. Sacks, and J. Tavantzis. "On the asymptotic behavior of solutions of certain quasilinear parabolic equations." Journal of Differential Equations 54, no. 1 (1984): 97-120.

[28] Pacard, F. "Partial regularity for weak solutions of a nonlinear elliptic equation." Manuscripta Mathematica 79, no. 2 (1993): 161-72.

[29] Passaseo, D. "New nonexistence results for elliptic equations with supercritical nonlinearity." Differential and Integral Equations 8, no. 3 (1995): 577-86.

[30] Passaseo, D. "Nontrivial solutions of elliptic equations with supercritical exponent in contractible domains." Duke Mathematical Journal 92, no. 2 (1998): 429-57.

[31] Perelman, G. "The entropy formula for the Ricci flow and its geometric applications." (2002): preprint arXiv:math/0211159.

[32] Rupflin, M. and M. Struwe. "Supercritical elliptic equations." Advanced Nonlinear Studies 12 (2012): 877-87; Addendum, Advanced Nonlinear Studies 13 (2013): 795-7.

[33] Stein, E. M. Harmonic Analysis: Real-Variable Methods, Orthogonality, and Oscillatory Integrals. Princeton Mathematical Series 43. Princeton, NJ: Princeton University Press, 1993.

[34] Struwe, M. "On the evolution of harmonic maps in higher dimensions." Journal of Differential Geometry 28, no. 3 (1988): 485-502.

[35] Struwe, M. Variational Methods. Applications to Nonlinear Partial Differential Equations and Hamiltonian Systems, 4th edn. Ergebnisse der Mathematik und ihrer Grenzgebiete 3. Folge. A Series of Modern Surveys in Mathematics 34. Berlin: Springer, 2008. 
[36] Troy, W. C. "The existence of bounded solutions of a semilinear heat equation." SIAM Journal on Mathematical Analysis 18, no. 2 (1987): 332-6.

[37] Weissler, F. B. "An $L^{\infty}$ blow-up estimate for a nonlinear heat equation." Communications on Pure and Applied Mathematics 38, no. 3 (1985): 291-5.

[38] Zou, H. "Symmetry of positive solutions of $\Delta u+u^{p}=0$ in $\mathbb{R}^{n}$." Journal of Differential Equations 120, no. 1 (1995): 46-88. 